\title{
Nonhuman primate models of human viral infections
}

\section{Jacob D. Estes ${ }^{1,2}$, Scott W. Wong ${ }^{2}$ and Jason M. Brenchley ${ }^{3 *}$}

Abstract | Humans have a close phylogenetic relationship with nonhuman primates (NHPs) and share many physiological parallels, such as highly similar immune systems, with them. Importantly, NHPs can be infected with many human or related simian viruses. In many cases, viruses replicate in the same cell types as in humans, and infections are often associated with the same pathologies. In addition, many reagents that are used to study the human immune response cross-react with NHP molecules. As such, NHPs are often used as models to study viral vaccine efficacy and antiviral therapeutic safety and efficacy and to understand aspects of viral pathogenesis. With several emerging viral infections becoming epidemic, NHPs are proving to be a very beneficial benchmark for investigating human viral infections.

Animal models of human viral infections are critical for understanding disease pathogenesis, vaccine modalities and therapeutic interventions in vivo. Ideally, studies of human viral infections would benefit from the ability to perform experiments using humans and samples from humans. Indeed, this is sometimes possible, as immune responses in humans can be measured before and after vaccination in peripheral blood, bronchoalveolar lavage, peripheral lymph nodes and the upper and lower gastrointestinal tract (via endoscopy). Moreover, vaccine development studies for certain infectious diseases can directly investigate the efficacy of the vaccine with pathogen challenge after vaccination (for example, Zika virus $(\mathrm{ZIKV})$ ). However, there are many important research questions that cannot directly be answered with experiments using human subjects; thus, animal models are required. Indeed, some infections result in high morbidity or mortality in humans; therefore, vaccine and/or challenge experiments in humans are not possible. Moreover, comparative observations that require substantial tissue volumes from multiple anatomical sites can negate the possibility of performing experiments in humans, especially if tissues are difficult to access. Finally, the outbred nature of the human population, coupled with demographic differences, creates substantial amounts of variation, which considerably complicates many research projects. Therefore, the development and detailed study of animal models for human diseases are absolutely critical.

Small animals, particularly rodents, are a mainstay of biological and immunological research. Indeed, studies in mice offer specific advantages: the ability to perform adoptive transfers of immune cells; the selective deletion of genes, embryonically or in specific cell types; the ability to control for MHC types ${ }^{1}$; selective colonization with controlled microbiota or removal of the microbiota ${ }^{2}$; the ability to intravitally image lymphocytes responding to antigens ${ }^{3,4}$; and the ability to create transgenic animals that express only specific antigen recognition receptors ${ }^{1}$. Moreover, small animals such as mice are fairly inexpensive to house, mature to adulthood rapidly, have short gestation periods and give birth to multiple offspring simultaneously. Indeed, the vast majority of our current knowledge of the immune system has, at some level, involved experiments with mice. Thus, Mus musculus is an extremely useful animal model for which the utility cannot be overstated.

Unfortunately, there are numerous biological phenomena that occur in humans that cannot be perfectly modelled in mice. Specifically, many viruses that cause disease in humans do not replicate in mice. Furthermore, human viruses that do replicate in mice may not result in the same types of pathologies that occur in humans. However, human viruses that are not well modelled in mice often do cause human-like disease in nonhuman primates (NHPs). Moreover, many of the reagents that are used to study immune responses in humans cross-react with NHPs (TABLE 1); as such, NHPs have become important animal models to study human viral infections (FIG. 1).

In this Review, we introduce species of NHPs that are experimentally used to recapitulate salient features of viral diseases in humans. We then discuss the immunological similarities and differences between NHPs and humans and the advantages and disadvantages of using NHP models of viral diseases (BOX 1). Finally, we consider several key examples of human viruses that have been experimentally modelled in NHP species. 
Table 1 | Nonhuman primate resources and Web databases

\begin{tabular}{|c|c|c|}
\hline Database & Website address & Information \\
\hline $\begin{array}{l}\text { NIH Office of Research Infrastructure } \\
\text { Programs - Comparative Medicine: } \\
\text { Vertebrate Models }\end{array}$ & $\begin{array}{l}\text { https://orip.nih.gov/ } \\
\text { comparative-medicine/programs/ } \\
\text { vertebrate-models }\end{array}$ & $\begin{array}{l}\text { Contains information pertaining to available vertebrate } \\
\text { models that are supported through the NIH Office of Research } \\
\text { Infrastructure Programs }\end{array}$ \\
\hline National Primate Research Centers & https://nprcresearch.org/primate/ & National resource for the scientific research community \\
\hline Biomedical Primate Research Centre & http://www.bprc.nl/en/home/ & $\begin{array}{l}\text { Primate research centre in the Netherlands that is committed } \\
\text { to using NHPs for crucial research when there are no suitable } \\
\text { alternatives }\end{array}$ \\
\hline NIH NHP Reagent Resource & $\begin{array}{l}\text { http://www.nhpreagents.org/NHP/ } \\
\text { default.aspx }\end{array}$ & $\begin{array}{l}\mathrm{NIH} \text {-supported resource to facilitate the optimal use of NHP } \\
\text { models in biomedical research. The resource identifies and can } \\
\text { provide specific validated reagents for in vitro and in vivo analysis }\end{array}$ \\
\hline NHP MHC web portal & $\begin{array}{l}\text { https://dholk.primate.wisc.edu/_ } \\
\text { webdav/dho/mhc_contract/web__. } \\
\text { portal/\%40files/prototype/index.html }\end{array}$ & $\begin{array}{l}\text { Contains information and protocols for genotyping and allele } \\
\text { discovery in macaques }\end{array}$ \\
\hline IPD-MHC database & $\begin{array}{l}\text { https://www.ebi.ac.uk/ipd/mhc/ } \\
\text { group/NHP }\end{array}$ & NHP database for MHC genes \\
\hline $\begin{array}{l}\text { Macaque Genotype and Phenotype } \\
\text { Database }\end{array}$ & https://mgap.ohsu.edu/ & $\begin{array}{l}\text { Provides access to genotype data collected on a large, pedigreed } \\
\text { rhesus macaque colony housed at the Oregon National Primate } \\
\text { Research Center }\end{array}$ \\
\hline $\begin{array}{l}\text { Baylor College of Medicine Human } \\
\text { Genome Sequencing Center - NHP } \\
\text { Genome Sequencing }\end{array}$ & $\begin{array}{l}\text { https://www.hgsc.bcm.edu/ } \\
\text { non-human-primates }\end{array}$ & $\begin{array}{l}\text { Sequence genomes from representative species from most of the } \\
\text { major branches of the phylogeny, including hominoids, Old World } \\
\text { monkeys, New World monkeys and lemurs }\end{array}$ \\
\hline \multirow{3}{*}{$\begin{array}{l}\text { NHP Reference Transcriptome } \\
\text { Resource }\end{array}$} & \multirow[t]{3}{*}{ http://nhprtr.org/ } & Phase I: RNA-seq analysis of tissue pools for individual NHP species \\
\hline & & Phase II: RNA-seq analysis of NHP tissue-specific transcriptomes \\
\hline & & Phase III: Tissue-specific small-RNA data for rhesus macaques \\
\hline Virus Pathogen Resource & https://www.viprbrc.org & $\begin{array}{l}\text { Database of genomic sequences of virus pathogen strains and } \\
\text { analysis tools }\end{array}$ \\
\hline
\end{tabular}

NHP, nonhuman primate; NIH, US National Institutes of Health; RNA-seq, RNA sequencing.

\section{Nonhuman primates as models}

For many different viruses, NHPs offer a window into the pathogenesis of human disease and into developing vaccine and therapeutic interventions aimed at treating and preventing viral infections (FIG. 1). Importantly, the innate and adaptive immune responses that NHPs elicit against viruses are, for the most part, very similar to human responses, meaning that these animals are more appropriate for some viral infections that cannot be modelled in mice. Moreover, many of the immunological reagents that are routinely used to identify human immunological molecules also cross-react with NHP molecules, which makes the transition to NHP studies less problematic, as information specific for NHPs is available on databases on the World Wide Web (TABLE 1).

\section{Old World monkey species}

Old World macaque NHP species are, by far, the most commonly used primate species for biomedical research. There are three species of Asian macaques that are commonly used for viral and/or immunological studies: Macaca mulatta (rhesus macaque), Macaca fascicularis (cynomolgus macaque), and Macaca nemestrina (pigtail macaque $)^{5-8}$. A wide variety of reagents (most of which are monoclonal antibodies against human proteins, which also cross-react with NHP antigens) are available to interrogate immunological responses to viruses, overt inflammation and immunological abnormalities. Moreover, viral epitope-loaded MHC tetramers exist for rhesus macaques $^{9-11}$ (at least three different MHC alleles) and pigtail macaques ${ }^{12}$ (at least one MHC allele) to study virus-specific $\mathrm{CD}^{+} \mathrm{T}$ cells (TABLE 1). Although there are no viral epitope-loaded MHC tetramers that are widely used for studies in cynomolgus macaques, the fairly limited MHC diversity of these animals makes them particularly attractive for studies involving allogenic responses and/or adoptive transfer experiments ${ }^{13-15}$.

Although rhesus, pigtail and cynomolgus macaques are very similar in genetic sequences and susceptibility to most of the viral infections we discuss herein, important differences exist that create particular nuances across these three species. For example, the type I interferoninduced, antiviral protein tripartite motif-containing protein 5 (TRIM5) has multiple genetic isoforms that have differing antiviral activity in each NHP species ${ }^{16}$. Rhesus macaques express heterogeneous TRIM5 genotypes, which differentially restrict simian immunodeficiency virus (SIV); however, pigtail macaques express only one TRIM5 genotype, which does not restrict $\mathrm{SIV}^{17}$. Of the three Asian macaque species typically used to study host and virus interactions, rhesus macaques are the most often studied, largely owing to their availability.

In addition to Asian macaques, several other Old World NHP species are employed for virology studies. In Africa, there are more than 50 species of NHPs that are natural hosts for SIV ${ }^{18}$. These species are often experimentally studied to understand how SIV has co-evolved with the natural hosts such that these NHP species do not develop simian AIDS (discussed below). The two 


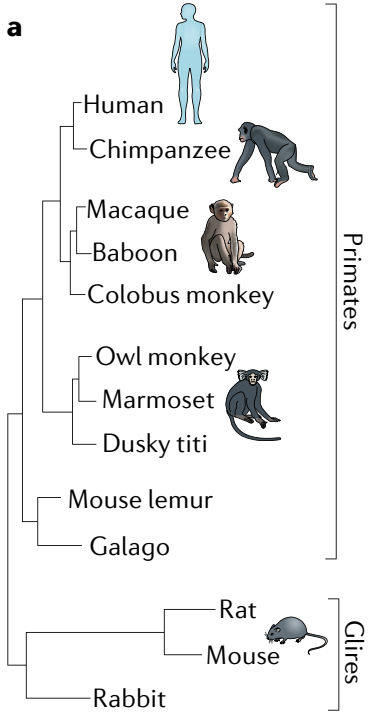

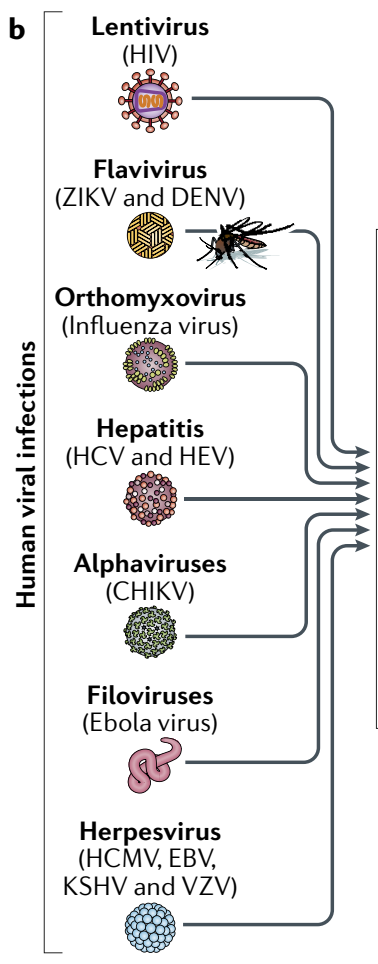

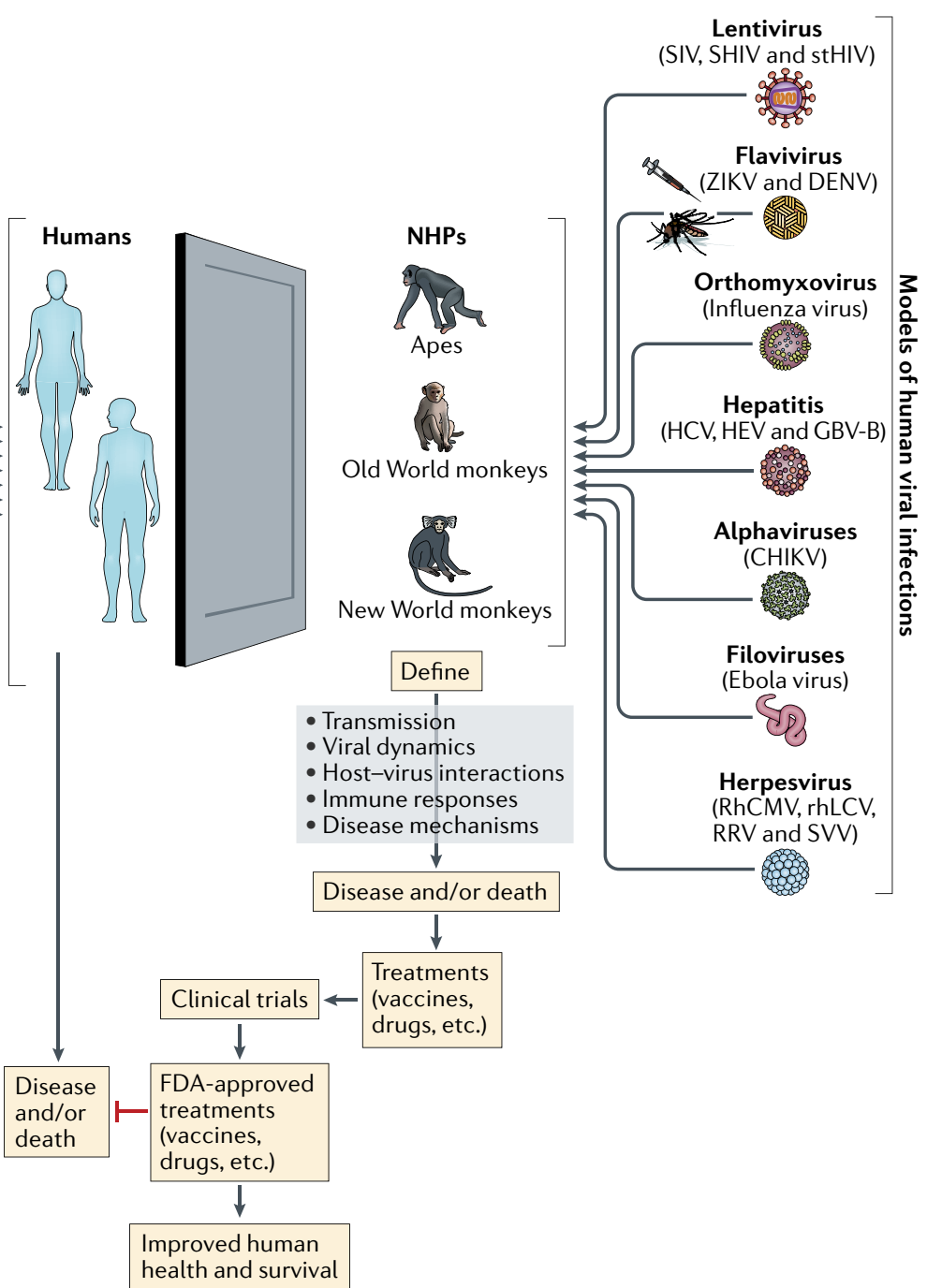

Fig. 1 | Nonhuman primate models to study viral diseases in humans. a |A phylogenetic tree demonstrating that nonhuman primates (NHPs) are the animals most closely related to humans, whereas mice are highly genetically distant. b | Many viruses that cause disease in humans also cause diseases in NHPs (Old World monkeys, New World monkeys or apes); alternatively, there are similar viruses that recapitulate the salient aspects of viral disease in humans. Thus, NHPs provide a valuable window into mechanisms of viral diseases and into developing novel therapeutic interventions to treat or vaccine modalities to prevent viral diseases. CHIKV, Chikungunya virus; DENV, dengue virus; EBV, Epstein-Barr virus; FDA, US Food and Drug Administration; GBV-B, hepatitis GB virus B; HCMV, human cytomegalovirus; HCV, hepatitis C virus; HEV, hepatitis E virus; KSHV, Kaposi's sarcoma-associated herpesvirus; LCV, lymphocryptovirus; RhCMV, rhesus CMV; RRV, rhesus macaque rhadinovirus; SHIV, simian HIV; SIV, simian immunodeficiency virus; stHIV, simian-tropic HIV; SVV, simian varicella virus; VZV, varicella zoster virus; ZIKV, zika virus. Part a is adapted with permission from REF. ${ }^{181}$, Cold Spring Harbor Laboratory Press.

most commonly used NHP SIV natural host species are sooty mangabeys (Cercocebus atys) and African green monkeys (Chlorocebus pygerythrus and Chlorocebus sabaeus $)^{18}$. However, ethical issues have limited the use of sooty mangabey subspecies (BOX 2), which confines the types of experiments that can be performed in all subspecies of Cercocebus atys. Therefore, the African green monkey Chlorocebus species have become a mainstay in natural host studies of SIV. In addition, African green monkeys can be infected with orthomyxoviruses ${ }^{19}$ (discussed below). Baboons (Papio sp.) are one of the more rarely used species of the Old World primates to study host and virus interactions ${ }^{20}$. These animals can be infected with many of the viruses discussed below; however, their availability and increased size (relative to other species) can prove limiting.

\section{New World monkey species}

Old World primates are the most frequently employed experimental primates for virology studies; however, New World primates, when appropriate, have distinct advantages. In particular, the marmosets (Callilthrix jacchus) are less expensive than the larger Old World primates and can be experimentally infected with several viruses that cause disease in humans (herpesviruses in particular, discussed below). Thus, it is more feasible to conduct studies that require larger numbers of animals than to conduct studies involving Old World primates. 


\section{Box 1 | The advantages and disadvantages of nonhuman primates over other models}

Viral infections have been modelled in mice and humans. However, nonhuman primate (NHP) models present an alternative approach with unique advantages and challenges.

\section{The advantages of NHP versus mouse models}

- Mice are not suitable for modelling many viral diseases owing to a lack of viral replication or inappropriate pathology

- NHP biology and physiology are most similar to those of humans

- Human-specific reagents can be used

- Many human viruses are overly sensitive to mouse type I interferon

- Disparate genotypes of NHPs better reflect the outbred human population

The disadvantages of NHP versus mouse models

- Increased cost and increased gestation period

- It is not possible to create gene-knockout or transgenic NHPs

- It is not possible to create germ-free NHPs or to perform parabiosis; experiments are limited to specific pathogen-free animals

- It is not possible to perform congenic animal studies

The advantages of NHP models versus human studies

- NHP and human immune systems are highly similar

- It is possible to perform longitudinal studies

- Environmental factors can be controlled

- Any anatomical site can be sampled

- The effects of additional infections can be studied

- Preventive vaccine or therapeutic efficacy can be studied with lethal viruses

- It is possible to deplete lymphocyte populations in NHPs in vivo

The disadvantages of NHP models versus human studies

- NHPs are not suitable for many viral diseases owing to a lack of viral replication or inappropriate pathology

- Some NHP genes exert antiviral activities differently than the equivalent human genes

- NHPs express a greater variety of MHC genes than humans

- NHP studies are limited to smaller numbers of animals

- Studies in NHPs are costly

- The genotypes of interferon-stimulated genes (for example, tripartite motif-containing protein 5 (TRIM5)) can influence antiviral responses

However, New World primate-specific reagents are not as abundantly available to interrogate host responses to viruses or viral-mediated pathogenesis.

\section{Ape species}

Ape species are evolutionarily more closely related to humans than are any other primate species (FIG. 1); thus, they are ideally suited as animal models aimed at studying phenomena that occur in humans. Indeed, experiments with chimpanzees were crucial for understanding the ontogeny of HIV, and chimpanzees are the only NHP species that support viral replication of HIV and hepatitis C virus (HCV). However, particular ethical concerns have dramatically limited the use of ape species as experimental animal models (BOX 2). In summary, NHPs can be appropriate animals to model many viral infections that cause diseases in humans. However, NHPs should be employed only when appropriate, and careful attention should be given to understanding the particular nuances and advantages that each NHP and viral species pair contains so that experiments may be designed appropriately.

\section{Immune responses in nonhuman primates}

In NHPs, immune responses to antigens, from both the adaptive and the innate arms of the immune system, are highly similar to those in humans. Although innate immune responses (which are commonly measured by expression patterns of interferon-stimulated genes) to viral infections are fairly widely conserved between NHPs and humans ${ }^{21-24}$, the most commonly measured immunological parameters in viral infections of primates are the magnitude and functionalities of virus-specific $\mathrm{T}$ cells and B cells.

The MHC region maps to chromosome 6 of humans; in rhesus macaques the MHC class I region is expanded and seemingly more complex. Humans contain one copy of the HLA-A, HLA-B and HLA-C genes per haplotype, whereas rhesus macaque and other macaque species can have multiple $M H C-A$ and $M H C-B$ genes per chromosome. Macaques can possess up to three $M H C$ - $A$ genes per haplotype. More is known about macaque $M H C-A$ genes than about the $M H C-B$ genes. The $M H C-A 1$ gene is the most polymorphic, whereas the other $M H C-A$ genes are more conserved and show lower levels of transcription. The $M H C-A 1$ gene is thought to encode MHC molecules that carry out classical peptide presentation whereas the other $M H C$ - $A$ genes are thought to share features with the nonclassical $M H C-I$ genes ${ }^{25,26}$. A similar increased diversity exists for NHP MHC-II alleles, compared 


\section{Box 2 | Special ethical considerations for nonhuman primate research}

All experiments that involve live animals require special ethical considerations. Given the close phylogenic similarities of nonhuman primates (NHPs) to humans, special concerns arise. Indeed, most animal care and use committees (ACUCs) set up specific review procedures to gauge the scientific validity of experiments that use NHPs, in addition to reviewing concerns related to the safety and well-being of animals enrolled in animal protocols.

Recently, the sooty mangabey subspecies Cercocebus atys lunulatus (the white-crowned mangabey) was added to the US Fish and Wildlife Service (FWS) list of endangered species. C. atys are natural hosts for certain strains of simian immunodeficiency virus (SIV). These animals exhibit nonprogressive infections with SIV and were the source of crossspecies transmission of the sooty mangabey strain of SIV ( SIV $_{\text {SMM }}$ ) into humans, which gave rise to HIV-2. However, because the $C$. atys lunulatus subspecies was put on the endangered list, it has considerably limited the types of experiments that can be performed in all subspecies of $C$. atys. This limitation is imposed despite the fact that one of the major atys subspecies (atys) is thriving in its natural environment and there are colonies of these animals at primate centres.

Chimpanzees also present specific considerations. These animals are genetically the most closely related NHPs to humans (FIG. 1) and, as such, they are highly relevant for studying viral diseases that affect humans. For example, a strain of SIV infects chimpanzees in the wild (SIV $\mathrm{CPZ}_{\text {) }}$; cross-species transmission of SIV ${ }_{\mathrm{CPZ}}$ gave rise to the HIV-1 epidemic, and chimpanzees are the only NHPs in which HIV-1 replicates efficiently in vivo. Moreover, chimpanzees were the goldstandard model for vaccine and pathogenesis studies of hepatitis $\mathrm{C}$ virus (HCV). Indeed, $\mathrm{HCV}$ does not replicate in other species of NHPs. However, in 2015, the FWS announced that it is categorizing captive chimpanzees as an endangered species, which are subject to legal protections. This announcement has almost negated research involving chimpanzees, substantially slowing progress on vaccine and antiviral therapeutic development.

to the equivalent human $H L A-D R, H L A-D P$ and HLA-DQ genes ${ }^{27}$.

Importantly, the canonical flow cytometric and intracellular cytokine staining modalities that are used for measuring antigen-specific $\mathrm{T}$ cell responses in humans are also meaningful for the analysis of virus-specific $\mathrm{T}$ cell responses in NHPs. Nucleated cells that express surface MHC from NHPs can be loaded with overlapping peptide pools that span pathogen open reading frames. Pathogen-specific T cells then become stimulated and produce effector cytokines that can be measured by standard enzyme-linked immunospot (ELISpot) assay or intracellular cytokine staining and flow cytometry ${ }^{22,28}$. Indeed, the number and variability of antigenspecific $\mathrm{T}$ cells that recognize individual $\mathrm{MHC}$-peptide complexes are quite similar in NHPs and humans, providing additional value to the NHP models.

The analysis of virus-specific B cells is also now possible in many species of NHPs. Traditionally, B cell responses were evaluated by removing $B$ cells from peripheral blood by negative selection and then assessing antibody production after antigen stimulation using ELISpot technology ${ }^{29}$. However, recent advances have used fluorochrome-conjugated viral antigens to isolate and analyse virus-specific B cells from multiple anatomical sites using flow cytometry. Moreover, the immunoglobulin locus of macaque species has recently been sequenced and studied in great detail ${ }^{30,31}$. Similar to the increased diversity of NHP MHCs, macaque immunoglobulin loci are substantially more diverse than in humans, with higher sequence diversity and copy-number variation. Although the antibody isotypes of NHP antibodies broadly reflect those found in both humans and mice, there are subtle differences, with NHPs having four immunoglobulin $\mathrm{G}$ ( IgG) isotypes. Moreover, the different isotypes bind to individual Fc receptors (such as CD16, CD32 and CD64) and complement similarly ${ }^{32}$. Thus, NHPs are a good model to understand antibody development against viruses in vivo.

\section{RNA viruses}

Numerous RNA virus families cause diseases in humans. RNA viruses can have a double-stranded or singlestranded genome, which may be negative or positive sense. Many of the RNA viruses that cause disease in humans induce similar diseases in NHPs, making NHPs phenomenal animal models.

\section{Lentiviruses}

HIV is responsible for the AIDS pandemic, and infection results in the progressive loss of $\mathrm{CD}^{+} \mathrm{T}$ cells and lymphoid tissue pathology.

The HIV-1 pandemic in humans resulted from cross-species transmissions of a strain of SIV $\left(\mathrm{SIV}_{\mathrm{CPZ}}\right)$ that infects central African chimpanzees (Pan troglodytes troglodytes). $\mathrm{SIV}_{\mathrm{CPZ}}$ is associated with increased mortality and AIDS-like immunopathology in wild-living chimpanzee communities and, in one case, a captive chimpanzee (reviewed in REF. ${ }^{33}$ ). Importantly, chimpanzees are the only NHP species that can be persistently infected with unmodified HIV-1; however, owing to their endangered status, the high costs of upkeep and the long duration of infection that is required to definitively assess AIDS development, chimpanzees are not a practical model for HIV infection and AIDS (BOX 2).

An extremely useful primate model of HIV is $\mathrm{SIV}_{\text {MAC }}$ (also known as $\mathrm{SIV}_{\mathrm{SMM}}$ ), which was first isolated from captive rhesus macaque monkeys with an AIDS-like illness in US primate centres ${ }^{34-36}$ (TABLES 1,2). SIV $_{\text {MAC }}$ was discovered to have arisen from a cross-species transmission between $\mathrm{SIV}_{\mathrm{SMM}}$-infected sooty mangabeys (the natural host, which do not develop disease) and rhesus macaques in captivity ${ }^{37,38}$. Importantly, SIV $_{\text {SMM }}$ from infected sooty mangabeys was also discovered to have established HIV-2 infection in humans ${ }^{39}$. Asian macaques are not natural hosts for primate lentiviruses and, when infected with certain strains of SIV, they develop a disease that resembles HIV-1 infection in humans. This discovery has led to the development of many SIV NHP models of HIV-1 infection. Collectively, these models have 
Table 2 | Key nonhuman primate models of human virus infections

\begin{tabular}{|c|c|}
\hline Human virus & Equivalent NHP virus ${ }^{a}$ \\
\hline \multicolumn{2}{|l|}{ NHP species: Asian macaque } \\
\hline \multirow[t]{3}{*}{ HIV-1 } & Simian immunodeficiency virus \\
\hline & Simian HIV \\
\hline & Simian-tropic HIV \\
\hline Dengue virus & Dengue virus \\
\hline Zika virus & Zika virus \\
\hline Ebola virus & Ebola virus \\
\hline Chikungunya virus & Chikungunya virus \\
\hline Varicella zoster virus & Simian varicella virus \\
\hline Cytomegalovirus & Rhesus cytomegalovirus \\
\hline Epstein-Barr virus & Rhesus lymphocryptovirus \\
\hline Kaposi's sarcoma-associated herpesvirus & Rhesus rhadinovirus \\
\hline Pox viruses & Monkeypox \\
\hline \multicolumn{2}{|l|}{ NHP species: marmoset } \\
\hline Hepatitis C & Hepatitis GB virus B \\
\hline
\end{tabular}

Transmitted/founder viruses The specific viral clone that initiates infection from a polyclonal infectious challenge swarm.

Simian HIVs

(SHIVs). Chimeric simian immunodeficiency viruses that encode portions of the HIV genome (generally

the envelope).

'Elite' controllers Individuals with protective MHC-I alleles that exert CD8 $T$ cell control of HIV or simian immunodeficiency virus viral replication. provided exceptional insight into fundamental aspects of HIV-1 transmission, prevention and pathogenesis.

Mucosal transmission. NHPs closely resemble humans in their physiology and immune system development ${ }^{40}$; thus, these animal models have been utilized extensively for lentiviral transmission studies. NHPs, primarily rhesus macaques, have been used for intravaginal, intrarectal, penile, oral and mother-to-child transmission studies ${ }^{41}$; these studies have imparted a better understanding of viral transmission across mucosal surfaces, the early host-viral dynamics within mucosal tissues, the first cells to be infected and the process of viral dissemination and establishment ${ }^{42-47}$.

Vaginal transmission studies in NHPs suggest that SIV initially infects antigen-presenting cells in the vagina, with the virus subsequently disseminating into genital-draining lymph nodes before spreading to proximal lymphoid nodes and finally systemically ${ }^{48}$. Subsequent vaginal challenge studies demonstrated that although macrophages and dendritic cells can be infected $\left(\mathrm{vRNA}^{+}\right)$, the vast majority of infected cells ( $\sim 90 \%)$ at the initial site of infection, in the lymph nodes and in other sites of dissemination were $\mathrm{CD} 4^{+} \mathrm{T}$ cells ${ }^{49}$. Importantly, the predominant infected $\left(\mathrm{vRNA}^{+}\right) \mathrm{CD}^{+}$ $\mathrm{T}$ cell population within the female genital tract at the early time points was demonstrated to be a population of HLA-DR' ${ }^{-}$and $\mathrm{Ki}^{-} 7^{-}$'resting' effector memory $\mathrm{T}$ cells ${ }^{49}$. This study also suggested that expanding viral replication in local $\mathrm{CD} 4^{+} \mathrm{T}$ cells at the mucosal portal of entry, which is fuelled by a robust innate inflammatory response to the virus, is required for the establishment of productive infection ${ }^{50}$. In addition, NHP vaginal transmission studies have highlighted the importance of resident and recruited $\mathrm{CD} 4^{+} \mathrm{T}$ cells in mucosal tissues for HIV mucosal transmission ${ }^{49,51}$.
NHP mucosal transmission experiments that use a high-dose inoculum have demonstrated the importance of the mucosal barrier as a 'bottleneck' to sexual transmission. In these studies, the initial high amount of viral RNA in the inoculum was reduced to very low levels of tissue-associated viral RNA shortly after challenge, suggesting that the virus initially gains access to a small number of susceptible target cells through vulnerable mucosal sites. Thus, there may be only small populations of founder-infected cells at the portal of entry ${ }^{51}$. Importantly, this finding was recapitulated using low-dose, repeatedchallenge transmission models via the rectal, vaginal or penile routes in rhesus macaques using distinct SIV isolates $\left(\mathrm{SIV}_{\text {MAC251 }} \text { and } \text { SIV }_{\text {SME } 660}\right)^{52}$. Many preclinical NHP vaccine and prevention studies now utilize the evaluation of the numbers of transmitted/founder viruses to determine efficacy, thus showing the important role that NHP models have played in delineating and recapitulating HIV-1 transmission $^{53-57}$.

Prevention. NHP models have been at the forefront of efforts to identify the types of immune responses needed for protection against HIV-1 and have become an accepted essential preclinical step for assessing vaccine strategies $^{58}$. Currently, the only HIV vaccine clinical trial that has demonstrated any protective efficacy was the RV144 trial, in which HIV-1 gp120 variable regions 1 and 2 (V1/V2)-specific IgG antibody titres were associated with some protection against HIV-1 acquisi$\operatorname{tion}^{59,60}$. Importantly, this finding was supported by studies in NHPs, as in macaques that were vaccinated with an 'RV144-like' immunization regimen, vaccineinduced protection from SIV acquisition was associated with non-neutralizing antibodies against gp120, which mapped to the V1/V2 region of the SIV envelope ${ }^{61}$. Additional evidence for the critical role of antibodies in protecting from HIV-1 transmission came from proofof-concept passive immunization NHP SIV studies ${ }^{62,63}$. Subsequent passive transfer experiments have demonstrated that certain HIV-specific neutralizing antibodies can provide complete protection against transmission of chimeric simian HIVs (SHIVs), which express HIV-1 envelope glycoproteins ${ }^{64-66}$.

The induction of broadly neutralizing antibodies (bNAbs) remains a major goal of HIV-1 vaccine development ${ }^{67}$. However, none of the previously tested HIV-1 vaccine candidates have been effective in eliciting bNAbs ${ }^{67}$. Because of the challenges in designing vaccine candidates that could induce bNAbs, alternative vaccine approaches were pursued, which focused on generating $\mathrm{CD}^{+}$cytotoxic T lymphocyte responses ${ }^{68}$. This approach was based, in part, on studies of 'elite' controllers; there was initial hope that powerful prime-boost vaccine approaches, which could greatly increase the magnitude and function of $\mathrm{CD}^{+} \mathrm{T}$ cell responses, would provide meaningful efficacy ${ }^{69}$.

Disease pathogenesis. Although SIV infection of Asian macaques (rhesus, cynomolgus and pigtail) is not identical to HIV-1 infection of humans, SIV infection of macaques does recapitulate key pathological features of HIV-1 infection, including $\mathrm{CD} 4^{+} \mathrm{T}$ cell 
tropism, progressive $\mathrm{CD} 4^{+} \mathrm{T}$ cell depletion, lymphoid tissue pathology, establishment of the latent reservoir, neuropathology and progression to AIDS $^{70}$. The range of both non-pathogenic and pathogenic NHP models utilized for HIV and AIDS research has authoritatively contributed to the discovery and establishment of key hallmarks of HIV-1 pathogenesis ${ }^{71}$.

Chronic immune activation and inflammation has emerged as one of the central features in HIV-1 pathogenesis ${ }^{72,73}$. Importantly, chronic immune activation is a hallmark of pathogenic HIV-1 infection in humans and SIV infection in macaques, whereas SIV chronically infected natural hosts (sooty mangabeys and African green monkeys) show an absence of immune activation $^{74}$. Studies in HIV-infected humans and SIVinfected NHPs demonstrate that HIV-associated or SIV-associated inflammation is a complex and multifactorial phenomenon that ultimately plays a major part in disrupting $\mathrm{T}$ cell homeostasis and driving lymphoid tissue pathology ${ }^{75,76}$. However, many studies have demonstrated that the elevation of microbial products (such as lipopolysaccharide (LPS), flagellin and bacterial DNA) that translocate from the damaged gastrointestinal tract into the systemic circulation of HIV-infected individuals may be a cause of chronic immune activation during progressive HIV-1 infection ${ }^{77-79}$. Moreover, studies in SIV-infected Asian macaques that capitalized on large tissue collections at necropsy demonstrated direct in situ evidence for microbial translocation into the gut parenchyma and subsequently into draining lymph nodes, liver and distal peripheral lymph nodes ${ }^{80}$, which does not occur in SIV-infected natural hosts ${ }^{77,80,81}$. Of note, a novel SIV-negative NHP model of experimental colitis demonstrated that gastrointestinal tract barrier damage was sufficient to drive local and systemic microbial translocation, inflammation and AIDS virus-like pathology, independently of SIV infection ${ }^{82}$. Collectively, human and NHP studies support a model of early structural and immunological damage to the gastrointestinal tract in progressive HIV-1 or SIV infections in humans and macaques, which leads to microbial translocation that contributes to persistent chronic immune activation.

Soon after initial studies described numerous histopathological changes to lymphoid tissue in HIV-infected humans, studies in SIV-infected NHPs also demonstrated similar pathology to HIV-infected humans, with parallel histological features such as lymphadenopathy with follicular hyperplasia to severe lymphoid depletion $^{83}$. Since these early reports, NHP models have proved to be an invaluable tool to elucidate the timing and mechanisms driving the progressive immunopathology of lymphoid tissue in HIV infection ${ }^{76}$. Studies in HIV-infected patients have demonstrated considerable lymphoid tissue damage associated with the deposition of fibrotic collagen ${ }^{84}$ - an observation that was subsequently confirmed in an NHP model of SIV infection, which linked fibrosis to immune activation and the induction of lymphocytes expressing transforming growth factor- $\beta$ (TGF $\beta)^{76}$. In addition, the rapid induction of a heterogeneous immunosuppressive regulatory $\mathrm{T}\left(\mathrm{T}_{\text {reg }}\right)$ cell response shortly after SIV infection in rhesus macaques recapitulates the reported increases and accumulation of $\mathrm{T}_{\text {reg }}$ cells and TGF $\beta$ expression in peripheral lymph nodes in HIV-infected humans ${ }^{85,86}$. Taken together, SIV infection of Asian macaques has recapitulated salient pathologies that occur in HIV-infected humans and has provided insights into novel therapeutic interventions and vaccine modalities to prevent HIV infection and improve the prognosis of HIV-infected humans.

\section{Hepatitis viruses}

Hepatitis viruses are important human pathogens that infect cells of the liver and cause liver damage (TABLE 2). The following section summarizes important NHP research on HCV and hepatitis E virus (HEV).

Hepatitis C virus. $\mathrm{HCV}$ is a blood-borne virus that can cause both acute and chronic hepatitis. Although there is currently no vaccine to prevent $\mathrm{HCV}$ infection, new US Food and Drug Administration (FDA)-approved direct-acting antivirals that target the HCV replication cycle have been demonstrated to cure more than $95 \%$ of $\mathrm{HCV}$-infected individuals. However, access to diagnosis and treatment remains low worldwide ${ }^{87,88}$. One substantial barrier to developing new, inexpensive and more potent treatments and an efficacious vaccine is the lack of a suitable animal model. Currently, chimpanzees are the only known animal species that it is possible to infect with HCV. However, because of the high costs, ethical concerns and regulatory restrictions (BOX 2), this NHP model has a very limited future ${ }^{89-91}$.

The NHP chimpanzee model played an instrumental role in the identification of HCV in 1989 and in advancing our understanding of $\mathrm{HCV}$ infection and disease, the study of immune responses to $\mathrm{HCV}$ and the pathogenesis of the disease $\mathrm{e}^{92}$. Numerous reports have demonstrated the importance of the innate (for example, type I IFNa and IFN $\beta$ responses) and adaptive (for example, $\mathrm{CD} 4^{+}$ and $\mathrm{CD}^{+} \mathrm{T}$ cell responses and $\mathrm{B}$ cell responses) arms of the immune system in the spontaneous resolution of acute HCV infection in chimpanzees, which confers long-lived immunity that considerably reduces the risk of reinfection ${ }^{92}$. One important caveat of the chimpanzee NHP model is that the natural course of HCV infection in chimpanzees is substantially different from that in humans ${ }^{93}$. Typically, HCV-induced hepatitis in chimpanzees is milder than in human HCV infection; however, severe hepatitis has been reported ${ }^{94}$. In addition, the progression to chronic hepatitis is much less common in chimpanzees, and in contrast to humans, liver cirrhosis is extremely rare ${ }^{95}$. Currently, it is unclear why the pathogenic course of HCV infection differs between chimpanzees and humans, but it may be owing, in part, to the lack of liver disease contributing cofactors (for example, alcohol consumption, obesity and a high-iron diet) in HCVinfected chimpanzees ${ }^{92}$. Alternatively, this difference may be a reflection of the low number of animals that have been followed in long-term studies to effectively evaluate the pathological consequences of long-term $\mathrm{HCV}$ infection; however, given the current US National Institutes of Health (NIH) policy to stop funding research in chimpanzees, the US Fish and Wildlife policy to restrict research in chimpanzees, and the limited resources 
available at national primate centres and Chimp Haven, where most $\mathrm{HCV}$-infected chimpanzees are located, it is unlikely that the differences in HCV infection between chimpanzees and humans will ever be fully understood ${ }^{92}$.

Although an alternative NHP HCV model remains elusive, hepatitis GB virus B (GBV-B), which is closely related to $\mathrm{HCV}$ and efficiently replicates in marmosets, represents a surrogate model for the study of HCV infection and disease ${ }^{96}$. The sequencing of GBV-B and the generation of a GBV-B molecular clone revealed important differences and similarities between GBV-B and HCV that has allowed for the construction of chimeric viruses ${ }^{96}$. Although GBV-B-HCV chimeric viruses do not perfectly recapitulate HCV biology, they do infect and replicate in New World NHPs and may serve as an important model for the development of HCV-targeting drugs that target specific regions of these chimeric viruses.

Hepatitis E virus. HEV is in the genus Orthohepevirus in the Hepeviridae family ${ }^{97}$ and shares many features with hepatitis A virus (HAV); however, HEV causes disease in humans and NHPs, whereas HAV does not cause disease in NHPs. The inoculation of NHPs with material from patients infected with water-borne non-A hepatitis was instrumental in the identification of $\mathrm{HEV}^{98,99}$. A number of NHP species (for example, chimpanzees, marmosets and macaques) are susceptible to experimental infection with HEV; however, cynomolgus macaques and rhesus macaques are the most commonly used species for studying HEV infection and disease $\mathrm{e}^{100-105}$. The severity of disease appears to be dependent on both the strain and the dose of virus used; however, cynomolgus macaques may be more sensitive to HEV infection, demonstrating more severe acute hepatitis than rhesus macaques or chimpanzees ${ }^{103,106,107}$.

As with HAV infection, damage to liver cells is thought to result more from the host immune response than from the direct effects of viral infection of hepatocytes $^{108}$. Histological changes in the liver of NHPs and patients are characteristic of acute hepatitis, including focal necrosis with lesions found in all regions of the lobule ${ }^{108}$. Similar to infection in humans, HEV infection in NHPs often leads to inflammation, consisting of Kupffer cells and polymorphonuclear leukocytes in focal lesions that are typical of hepatitis ${ }^{108}$. In contrast to humans, pregnancy does not exacerbate the disease course in cynomolgus macaques ${ }^{104}$. Importantly, NHP models have been extensively used for the preclinical testing of vaccines against $\mathrm{HEV}$, and a vaccine to prevent HEV infection has been developed but is not yet available worldwide ${ }^{109-114}$.

Kupffer cells

Specialized liver-resident macrophages involved in recycling iron from dying red blood cells and in phagocytosis of microbial antigens that drain into the liver from the portal vein.

Guillain-Barre syndrome Rare autoimmune disease associated with neurological symptoms that can lead to paralysis. use mice deficient in type I interferon signalling ${ }^{116-118}$. Thus, the mouse model of flavivirus infection may not recapitulate all of the salient aspects of virus and host interactions that occur in vivo in humans. As such, NHPs have become an important model to study these important viral infections.

Dengue virus. Given the enormous public health concerns associated with DENV infection (TABLE 2), it is important to develop animal models that recapitulate key features of human infection ${ }^{119}$. Wild-type mice are not susceptible to DENV infection; thus, considerable effort has been made to identify appropriate NHP mod$\mathrm{els}^{119}$. There are at least four serotypes of DENV, each of which shares approximately $65 \%$ genetic similarity with the other three, which further complicates the modelling of DENV infection in NHPs ${ }^{120}$. Moreover, there is substantial evidence that preinfection with one serotype of DENV can result in more severe disease upon challenge with another DENV serotype, which may be facilitated by weakly cross-reacting antibodies binding the different serotypes ${ }^{120}$. Thus, ideally, several serotypes of DENV would need to be capable of replicating and causing disease in NHPs. Many strains of DENV, routes of infections, sources of virus and species of NHPs have been tested ${ }^{119,121}$. The most commonly studied NHPs for DENV infections are macaque species, Old World patas monkeys and African green monkeys ${ }^{119,121}$. All of the serotypes of DENV that have been tested (DENV 1-4) replicate to high levels (up to 100 million copies per millilitre of plasma), and viraemia lasts for approximately 1 week, which is similar to infection in humans. However, NHPs do not seem to manifest the same types of symptoms that are observed in DENV-infected humans ${ }^{119,121}$.

NHPs have been employed to measure vaccineinduced immunity against DENVs and to ensure that attenuated viral vaccines lack the ability to replicate to high levels in vivo ${ }^{121,122}$. Many vaccine modalities that have been shown to be safe in NHPs and that induce high titres of neutralizing antibodies against DENV have been advanced to human clinical trials ${ }^{121,123,124}$, and one has even shown protection after human challenge with $\mathrm{DENV}^{124}$.

Zika virus. ZIKV was first identified as a human pathogen in Africa in 1952, but the first human epidemic was recognized in Yap Island in 2007, which spread to a larger epidemic in French Polynesia in 2013-2014 (REF. ${ }^{125}$ ). Subsequently, ZIKV has spread widely in warmer climates, with infections numbering in the tens of thousands and coinciding with mosquito life cycles ${ }^{125}$. ZIKV infections are generally not life-threatening; however, infection is associated with a rare chance of Guillain-Barre syndrome. In addition, ZIKV infection of pregnant women has been linked with birth defects.

Several studies have employed NHPs (cynomolgus macaques, rhesus macaques and pigtail macaques) to study salient features of ZIKV pathogenesis ${ }^{126-129}$ (TABLE 2). In general, ZIKV infection of NHPs is facilitated by subcutaneous injection, which models infection via mosquito bite, and recapitulates many features of human 
infection, including mild weight loss, slight fever and elevated liver enzymes ${ }^{126-129}$. NHPs infected with ZIKV became viraemic, and plasma viraemia peaked between 2 and 6 days after infection; subsequently, these animals seemed to clear plasma viraemia within 10 days ${ }^{126-129}$. ZIKV was detected in semen, vaginal fluid, urine, saliva, cerebrospinal fluid and lymphoid tissue as well as in the gastrointestinal tract and the central nervous system (CNS) after the resolution of viraemia in the blood ${ }^{127,128}$. Finally, NHPs infected with ZIKV mount virus-specific humoral and cellular immune responses, which protect against subsequent homologous and heterologous challenges, suggesting that NHPs are an appropriate animal model to study vaccine-mediated protection ${ }^{22,127,128}$. Indeed, multiple vaccine modalities have been tested in NHPs, which induce the production of neutralizing antibodies against ZIKV and are protective against subsequent challenge ${ }^{130-132}$. Having shown protection in NHPs, many of these vaccines are moving forwards into human clinical trials.

One of the most worrisome aspects of ZIKV infection is the apparent ability of ZIKV to cross the placenta during pregnancy and infect the developing fetus. Indeed, ZIKV infection during pregnancy is associated with fetal developmental abnormalities leading to microcephaly. An animal model that recapitulates this phenomenon would be important for developing therapeutic interventions. Pregnant rhesus macaques infected with ZIKV had viraemia lasting up to 55 days, which was far longer than in nonpregnant animals and similar to what has been reported in pregnant women ${ }^{127,133}$. Importantly, in pregnant pigtail macaques infected with ZIKV, the fetus showed reduced brain growth, neuronal damage, white matter deficiency and gliosis, and the virus was detected in the placenta and fetal brain and liver ${ }^{134}$. Finally, recent work has suggested that ZIKV RNA can persist in the tissues of NHPs for prolonged periods of time after the resolution of viraemia ${ }^{22,135}$. Thus, NHPs may represent an appropriate animal model to understand the mechanisms of maternal-fetal ZIKV transmission, pathogenesis and persistence and to develop vaccines and/or therapeutic interventions.

West Nile virus. Multiple NHP species have been experimentally infected with WNV. WNV is spread by mosquitoes, and infection in humans is usually asymptomatic; however, $20 \%$ of infected humans may have fevers, aches, vomiting, diarrhoea or rash. Moreover, $\sim 0.5 \%$ of WNV-infected humans have more severe disease with CNS involvement, and paralysis or death are possible ${ }^{136}$. As WNV is a biohazard level 3 pathogen, special precautions are required and, as such, not all primate facilities are capable of working with the virus. Rhesus macaques and marmosets can be experimentally infected with $\mathrm{WNV}$, and both replicate the virus in vivo to high titres; however, marmosets show higher viral titres and more extensive tissue penetrance ${ }^{137}$. Although both species demonstrated adaptive and innate immune responses to WNV, neither had any clinical signs of WNV infection, which is similar to the majority of WNV-infected humans ${ }^{137}$. Notably, intrathalamic infection of rhesus macaques led to viral spread throughout the CNS without any clinical symptoms of disease ${ }^{138}$. It is unclear whether WNV fever or neurological disease occurs in NHPs, and very large numbers of animals would likely be required to ascertain this. However, NHPs may represent an appropriate model to study vaccine-mediated protection against WNV infection, considering that they replicate the virus and mount antibody and cellular immune responses. Accordingly, several vaccine modalities against WNV have already been used in NHPs; some of these seem to protect NHPs from subsequent WNV challenge and have entered trials in humans ${ }^{139-142}$.

\section{DNA viruses}

There are five families of DNA viruses that infect humans: Adenoviridae, Herpesviridae, Papoviridae, Parvoviridae and Poxviridae. Of these, the Herpesviridae and Poxviridae are associated with many important diseases in humans, whereas the Papoviridae are associated with rare pathological conditions, and the Adenoviridae and Parvoviridae, which are rarely associated with human disease, are being developed for heterologous gene delivery.

\section{Herpesvirus}

Herpesviruses are a family of large, enveloped DNA viruses that have linear, double-stranded DNA genomes and that infect almost all animal species. Here, we describe studies of simian herpesviruses (which are closely related to human herpesviruses) that aim to elucidate mechanisms of pathogenesis, to define the host immune response to control latent herpesvirus infections and to identify the viral and host factors that lead to virus reactivation.

Alpha-herpesvirus. Human varicella zoster virus (VZV; TABLE 2) is an alpha-herpesvirus that causes chickenpox and herpes zoster in humans. An attenuated strain of VZV has been licensed as a vaccine and is effective at preventing chickenpox, but is less effective at preventing VZV reactivation (herpes zoster) in elderly and immunocompromised individuals. Unfortunately, VZV exhibits strict species specificity, which prevents animal infection studies to elucidate the mechanisms of herpes zoster disease. Thus, an animal model that closely parallels key features of VZV infection and pathogenesis is required.

Simian varicella virus (SVV) is a related virus that was isolated in the 1960s; SVV is morphologically and genetically similar to VZV, and several groups have reported that inoculation of Old World monkeys (African green monkeys and cynomolgus macaques) can lead to varicella with or without the establishment of latency (reviewed in REF. ${ }^{143}$ ). Importantly, intrabronchial inoculation of rhesus macaques with SVV appears to recapitulate all the essential features of VZV infection in humans, including latency. Furthermore, this model has enabled researchers to determine how the antibody-mediated depletion of specific immune cells affected primary SVV infection. One group found that depletion of $\mathrm{CD}^{+} \mathrm{T}$ cells during a primary SVV infection led to widely disseminated varicella accompanied by higher and prolonged viral loads compared with animals depleted of $\mathrm{CD}^{+} \mathrm{T}$ cells and of 
$\mathrm{B}$ cells ${ }^{144}$. This finding implies that $\mathrm{CD} 4^{+} \mathrm{T}$ cell responses may be important for the control of varicella or herpes zoster disease in humans.

Several attempts have been made to reproducibly induce SVV reactivation in macaques that have an established latent infection. The most recent study utilized full-body irradiation in addition to treatment with prednisone and tacrolimus (both are immunosuppressive drugs) and was successful in reactivation in four of four rhesus macaques. Surprisingly, a control animal also developed a rash, implying that the stress associated with the transport of animals to the site of irradiation was sufficient to induce reactivation ${ }^{145}$. Taken together, the NHP model of VZV infection has provided important information related to immunological pressure and mechanisms of viral reactivation from latency, including how immunodeficiency can lead to viral reactivation. Moreover, this model will be useful in advancing vaccines aimed at reducing the incidence of herpes zoster.

Beta-herpesvirus infections: rhesus cytomegalovirus. Human cytomegalovirus (HCMV; strictly tropic to humans) is an important pathogen with widespread seropositivity in humans. NHPs are hosts to homologues of HCMV (TABLE 2), and several CMV isolates have been obtained and sequenced from numerous NHPs, including chimpanzees, baboons, cynomolgus macaques and other Old World monkeys. The morphology and genome sequences of NHP CMV isolates are quite similar across species and reveal a close evolutionary relationship with their hosts. The most widely studied NHP CMV is rhesus CMV (RhCMV), of which two isolates are utilized for pathogenesis and vaccine studies (reviewed in REF. ${ }^{146}$ ).

Our current fundamental understanding of rhesus macaque cellular immunity originated from studies characterizing ex vivo $\mathrm{CD}^{+}$and $\mathrm{CD} 8^{+} \mathrm{T}$ cell subsets from RhCMV-infected animals ${ }^{147}$. These early studies defined the key immunological markers on $\mathrm{CD}^{+}$and $\mathrm{CD} 8^{+}$naive and memory (central and effector subsets) $\mathrm{T}$ cells and performed comprehensive functional analyses of these populations. This immunological information led to a continued and expanded use of the rhesus macaque model for investigations into viral pathogenesis and vaccine development.

Some of the most intriguing studies of RhCMV in rhesus macaques involve using RhCMV as a vaccine vector to prevent SIV infection and disease. Here, investigators found that RhCMV vectors, particularly rhCMV68.1, which encoded SIV antigens, were capable of infecting CMV-naive and CMV-seropositive rhesus macaques and eliciting persistent $\mathrm{SIV}$-specific $\mathrm{CD} 4^{+}$and $\mathrm{CD}^{+} \mathrm{T}$ effector memory $\left(\mathrm{T}_{\mathrm{EM}}\right)$ cell responses ${ }^{148}$. SIV-specific $\mathrm{CD} 8^{+} \mathrm{T}$ cells were demonstrated to be MHC class I-restricted and were also restricted by MHC class II or non-polymorphic MHC-E, implying that the SIV RhCMV vaccine elicited responses from unconventional $\mathrm{CD}^{+} \mathrm{T}$ cells ${ }^{28,149}$. Importantly, $\mathrm{T}_{\mathrm{EM}}$ cell responses were present at extralymphoid sites in vaccinated animals and were effective at preventing progressive SIV infection after challenge with $\mathrm{SIV}_{\mathrm{MAC239}}$. Moreover, an analysis of vaccinated animals revealed that protection from progressive SIV infection was correlated with the magnitude of the peak SIV-specific
$\mathrm{CD}^{+} \mathrm{T}$ cell response. Interestingly, these protected animals exhibited effective SIV control even after antibodymediated depletion of $\mathrm{CD} 4^{+}$and $\mathrm{CD} 8^{+} \mathrm{T}$ cells, implying that the vaccine was capable of clearing SIV infection ${ }^{150}$. That RhCMV-based vaccines against SIV have led to such impressive control against pathogenic SIV infection has led to ongoing studies using similar human CMV-vectored approaches being developed for human trials.

Gamma-1-herpesviruses: lymphocryptoviruses. Epstein-Barr virus (EBV; TABLE 2) is a gamma-1herpesvirus or lymphocryptovirus (LCV) that infects most humans by the time they reach adulthood. EBV is strictly human-tropic, which makes studies of viral pathogenesis extremely difficult in animals.

Multiple NHP species from Old World and New World monkeys harbour related LCVs, with each LCV capable of immortalizing B cells from their natural host, which is a key feature of human EBV infection (reviewed in REF. ${ }^{151}$ ). The most widely studied NHP LCV is rhesus LCV (rhLCV), which was originally isolated from a lymphoblastoid cell line that was established from a malignant lymphoma that developed in a rhesus macaque. Importantly, several publications have reported that rhLCV is associated with diseases that parallel EBV, including B cell lymphomas and hairy leukoplakia in SIV-infected macaques. In addition, primary rhLCV infection in rhesus macaques parallels primary EBV infection in humans ${ }^{151}$. The utility of the rhLCV rhesus macaque model is further underscored by the creation of a novel rhLCV BACmid, which can produce infectious virus that is capable of immortalizing B cells from rhesus macaques ${ }^{152}$. The rhLCV BACmid system enables the interrogation of EBV-expressed genes involved in viral pathogenesis and for development of novel vaccine approaches.

Immunologically, rhLCV infection of rhesus macaques closely parallels EBV infection in humans. A detailed study of naturally rhLCV-infected animals revealed that the humoral response develops against multiple viral antigens. Animals exhibited hierarchical responses, with the more robust humoral response to late viral proteins and less frequent responses to early and immediate-early proteins. Although a direct comparison could not be made between rhesus macaque humoral responses and human sera responses, the macaque responses still correlated well with EBV-infected human samples ${ }^{153}$.

$\mathrm{T}$ cell-specific immunity to rhLCV has also been examined. An exhaustive study compared naturally rhLCV-infected rhesus macaques and EBV-infected humans and challenged both species with a recombinant vaccinia virus encoding individual EBV antigens; the results revealed that similar $\mathrm{T}$ cell responses develop to late antigens in both humans and macaques as persistent infection progresses over time. The close correlations in humoral and $\mathrm{T}$ cell-specific responses that are shared between rhesus macaques and humans will allow future vaccine evaluation trials using the rhLCV rhesus macaque model (for example, the phase II clinical trial in humans vaccinated with soluble EBV gp350 (Ref. ${ }^{154,155)}$ ). Related studies in rhesus macaque inoculated with a recombinant rhLCV (encoding EBV gp350 in place of rhLCV gp350) 
revealed that EBV gp350 could functionally substitute for rhLCV gp350, as the recombinant rhLCV could infect animals as effectively as wild-type rhLCV ${ }^{156}$. This finding demonstrates that the rhLCV rhesus macaque model is an excellent system to develop and evaluate novel vaccine approaches to prevent EBV-associated diseases, including infectious mononucleosis ${ }^{156-158}$.

Gamma-2 herpesviruses: rhadinoviruses. Kaposi's sarcoma-associated herpesvirus (KSHV; TABLE 2) is a gamma-2 herpesvirus or rhadinovirus ${ }^{159}$ and the aetiological agent of several rare cancers, including Kaposi's sarcoma, primary effusion lymphoma ${ }^{160}$ and multicentric Castleman disease ${ }^{161}$. Unfortunately, KSHV displays strict tropism for humans, with the exception of one report of KSHV infection and Kaposi's sarcomalike lesions in common marmosets ${ }^{162}$, a model that is being further developed. The lack of a direct animal model of KSHV has led to the development of an NHP model that uses the closely related rhesus macaque rhadinovirus (RRV; a gamma-2-herpesvirus) ${ }^{163}$. Two independent strains of RRV have been isolated ${ }^{163,164}$ that exhibit different pathogenic potential. Strain H26-95 is less pathogenic than strain 17577, as strain H26-95 has not been associated with disease, even in SIV-infected animals ${ }^{165}$. By contrast, strain 17577 is associated with B cell lymphoma and a mesenchymal proliferative lesion, referred to as retroperitoneal fibromatosis, that resembles Kaposi's sarcoma in SIV-infected rhesus macaques ${ }^{166}$. The difference in pathogenic potential has led investigators in different research directions as to how to best utilize the RRV model for human virus infection studies (reviewed in REF. ${ }^{167}$ ).

Immunologically, RRV infection can induce robust innate and virus-specific humoral and cell-mediated responses in rhesus macaques. This finding was demonstrated when naive rhesus macaques were experimentally infected with a recombinant RRV lacking the eight viral interferon regulatory factors (vIRFs; $\mathrm{RRV}_{\text {vIRF-ko }}$ ) or wild-type RRV. Animals infected with $\mathrm{RRV}_{\text {viRF-ko }}$ had earlier and sustained production of pro-inflammatory cytokines and earlier induction of RRV-specific T cell responses than the wild-type RRV-infected animals. Humoral responses were essentially identical. The altered host response to $\mathrm{RRV}_{\text {vIRF-ko }}$ infection was associated with decreased viral loads and diminished $\mathrm{B}$ cell hyperplasia, implying that the vIRFs function to slow the innate and adaptive immune responses in animals ${ }^{168}$. Because KSHV also expresses vIRFs, these data point to the importance of innate immunity against the human virus and to potential therapeutic interventions that might inhibit vIRFs.

\section{Conclusions and perspective}

Here, we provide an overview of many of the important human viruses that can be effectively modelled in NHPs although it is outside the scope of this Review to cover all human viral pathogens (see BOX 3 for a brief mention of several other notable examples of human viral pathogens). NHPs represent essential animal

\section{Box 3 | Emerging viruses that can be modelled in nonhuman primates}

\section{Filoviruses}

Filoviruses are filamentous, single-stranded, negative-sense RNA viruses. Some members of this family (in particular, Ebola virus and Marburg virus) can cause severe viral haemorrhagic fevers with very high mortality. Viral-induced inflammation is thought to result in much of the pathology observed in infected individuals, with vascular permeability and organ failure occurring in upwards of $70 \%$ of infected individuals. Asian macaques can be infected with Ebola and Marburg viruses, and the pathology closely resembles that found in humans, although the mortality might be slightly higher in nonhuman primates (NHPs) than in humans, with near $100 \%$ mortality in $\mathrm{NHPs}^{169}$. NHPs have been used to test the therapeutic capacity of antiviral compounds against filoviruses and the ability of different vaccine modalities to protect against subsequent viral challenge ${ }^{170-173}$. Many of these modalities are being tested in humans to treat and prevent future filovirus epidemics.

\section{Pox viruses}

Although smallpox virus (TABLE 1) has been eradicated, there are concerns that it could be weaponized. In addition, monkeypox (MPXV) virus infections of humans lead to symptoms that are similar to smallpox, with fever, weight loss and lesions, and up to $10 \%$ mortality. Thus, there is interest in developing animal models of pox virus infection. MPXV infection of rhesus macaques also results in disease pathology that closely resembles smallpox infection of humans; the severity of symptoms and the overall lethality vary by the dose and route of virus challenge, and NHPs have been used to test vaccine modalities and antivirals ${ }^{174-176}$.

\section{Chikungunya virus}

Chikungunya virus (CHIKV) can infect both rhesus macaques and cynomolgus macaques. Moreover, similar to humans, aged macaques have higher and more prolonged viraemia than younger animals ${ }^{177,178}$. In addition, Asian macaques become febrile during acute infection, and some animals also experience rash and joint swelling (especially if higher doses of virus are used for infections), which are also similar to human infection symptoms. After infection, CHIKV can be detected in the liver, lymphoid tissue, synovial and skeletomuscular tissues, lungs and kidneys. These sites can also manifest some degree of structural abnormalities and lymphocyte infiltration. CHIKV infection of Asian macaques can also lead to neurological abnormalities, with symptoms of meningoencephalitis and leukocytes in cerebrospinal fluid. Adaptive immune responses are evident in NHPs, with proliferation of T cells and induction of T cell responses directed against multiple CHIKV protein epitopes. During infection, NHPs also induce B cell responses to CHIKV against numerous viral proteins, the antibodies produced include multiple isotypes, and by 30 days postinfection, antibodies are able to neutralize CHIKV in vitro. Thus, Asian macaques represent a viable animal model to study vaccines and therapeutics to prevent CHIKV infection and/or reduce symptoms associated with infection ${ }^{179,180}$. 
models to study many viral infections that affect human health, in a controlled setting, where strict adherence to protocols and/or drug regimens is enforced to ensure reproducibility. These studies include virological and immunological analyses and interventional vaccine and therapeutic strategies aimed at treatment and prevention. Longitudinal analyses, coupled with the ability to sample any anatomical site in animal models, provide distinct capabilities that are not possible in humans. NHP studies are highly regulated at the local and national levels, which ensures animal welfare and wellbeing. In summary, NHPs are a valuable and reliable animal model.

Data from NHP studies have provided invaluable insights into human viral infection, improving our understanding of mechanisms of disease pathogenesis, innate and adaptive immune responses and vaccine and therapeutic modalities. Studies from NHPs have guided numerous vaccine and therapeutic trials in humans, including ZIKV vaccine trials. Future work might involve determination of how modulation of the commensal microbiota influences viral pathogenesis, novel strategies for viral prevention and eradication, the direct effect of how immune responses and/or host genetics affect susceptibility to viral infections and their outcome, novel approaches to diagnose and treat viral diseases (including virusmediated cancers), testing novel combination (virustargeted and host-targeted) approaches to treatment and the development of multipathogen models to better reflect regions in the world that have a high burden of pathogens.

Published online 19 March 2018
1. Zehn, D. \& Wherry, E. J. Immune memory and exhaustion: clinically relevant lessons from the LCMV model. Adv. Exp. Med. Biol 850, 137-152 (2015).

2. Belkaid, Y. \& Harrison, O. J. Homeostatic immunity and the microbiota. Immunity 46, 562-576 (2017).

3. Hickman, H. D. New insights into antiviral immunity gained through intravital imaging. Curr. Opin. Virol 22, 59-63 (2017)

4. Mueller, S. N. \& Hickman, H. D. In vivo imaging of the $\mathrm{T}$ cell response to infection. Curr. Opin. Immunol. 22, 293-298 (2010).

5. Rimmelzwaan, G. F. et al. Induction of protective immunity against influenza virus in a macaque model: comparison of conventional and iscom vaccines. J. Gen. Virol 78, 757-765 (1997).

6. Klatt, N. R. et al. Compromised gastrointestinal integrity in pigtail macaques is associated with increased microbial translocation, immune activation, and IL-1 7 production in the absence of SIV infection. Mucosal Immunol 3, 387-398 (2010).

7. Baskin, C. R. et al. Integration of clinical data, pathology, and cDNA microarrays in influenza virusinfected pigtailed macaques (Macaca nemestrina). J. Virol. 78, 10420-10432 (2004).

8. Ericsen, A. J. et al. Microbial translocation and inflammation occur in hyperacute immunodeficiency virus infection and compromise host control of virus replication. PLoS Pathog. 12, e 1006048 (2016).

9. Mudd, P. A. et al. Escape from $\mathrm{CD}^{+} \mathrm{T}$ cell responses in Mamu-B*00801+ macaques differentiates progressors from elite controllers. J. Immunol. 188, 3364-3370 (2012)

10. Price, D. A. et al. Public clonotype usage identifies protective Gag-specific CD8+ T cell responses in SIV infection. J. Exp. Med. 206, 923-936 (2009).

11. Minang, J. T. et al. The Mamu B 17-restricted SIV Nef IW9 to TW9 mutation abrogates correct epitope processing and presentation without loss of replicative fitness. Virology 375, 307-314 (2008).

12. Klatt, N. R. et al. Dynamics of simian immunodeficiency virus SIVmac239 infection in pigtail macaques. J. Virol. 86, 1203-1213 (2012).

13. Karl, J. A. et al. Major histocompatibility complex haplotyping and long-amplicon allele discovery in cynomolgus macaques from Chinese breeding facilities. Immunogenetics 69, 211-229 (2017).

14. Ericsen, A. J. et al. Whole genome sequencing of SIVinfected macaques identifies candidate loci that may contribute to host control of virus replication. Genome Biol. 15, 478 (2014).

15. Greene, J. M. et al. Allogeneic lymphocytes persist and traffic in feral MHC-matched mauritian cynomolgus macaques. PLOS ONE 3, e2384 (2008)

16. Wu, F. et al. TRIM5alpha restriction affects clinical outcome and disease progression in simian immunodeficiency virus-infected rhesus macaques. J. Virol. 89, 2233-2240 (2015)

17. Yu, C. Q. et al. The TRIMCyp genotype in four species of macaques in China. Immunogenetics 65, 185-193 (2013).
18. Sodora, D. L. et al. Toward an AIDS vaccine: lessons from natural simian immunodeficiency virus infections of African nonhuman primate hosts. Nat. Med. 15, 861-865 (2009)

19. Matsuoka, Y. et al. African green monkeys recapitulate the clinical experience with replication of live attenuated pandemic influenza virus vaccine candidates. J. Virol. 88, 8139-8152 (2014)

20. Locher, C. P. et al. Baboons as an animal model for human immunodeficiency virus pathogenesis and vaccine development. Immunol. Rev 183, 127-140 (2001).

21. Harris, L. D. et al. Downregulation of robust acute type I interferon responses distinguishes nonpathogenic simian immunodeficiency virus (SIV) infection of natural hosts from pathogenic SIV infection of rhesus macaques. J. Virol. 84, 7886-789 (2010).

22. Aid, M. et al. Zika virus persistence in the central nervous system and lymph nodes of rhesus monkeys. Cell 169, 610-620.e4 (2017). This study used ZIKV-infected NHPs to analyse cells that were targeted by the virus in vivo and to carefully examine virus-mediated pathogenesis and long-term sanctuaries of virus-infected cells.

23. Jacquelin, B. et al. Nonpathogenic SIV infection of African green monkeys induces a strong but rapidly controlled type I IFN response. J. Clin. Invest. 119, 3544-3555 (2009).

24. Robinson, B. A., Estep R. D. Messaoudi, I. Rogers, K. S. \& Wong, S. W. Viral interferon regulatory factors decrease the induction of type I and type II interferon during rhesus macaque rhadinovirus infection. J. Virol. 86, 2197-2211 (2012).

25. de Groot, N., Doxiadis, G. G., Otting N. de Vos-Rouweler, A. J. \& Bontrop, R. E. Differential recombination dynamics within the $\mathrm{MHC}$ of macaque species. Immunogenetics $66,535-544$ (2014).

26. Doxiadis, G. G. et al. Haplotype diversity generated by ancient recombination-like events in the MHC of Indian rhesus macaques. Immunogenetics 65 , 569-584 (2013)

27. Otting, N. et al. The orthologs of HLA-DQ and -DP genes display abundant levels of variability in macaque species. Immunogenetics 69, 87-99 (2017).

28. Hansen, S. G. et al. Broadly targeted $C D 8^{+} \mathrm{T}$ cell responses restricted by major histocompatibility complex E. Science 351, 714-720 (2016).

29. Klatt, N. R. et al. SIV infection of rhesus macaques results in dysfunctional T- and B-cell responses to neo and recall Leishmania major vaccination. Blood 118, 5803-5812 (2011).

30. Ramesh, A. et al. Structure and diversity of the rhesus macaque immunoglobulin loci through multiple de novo genome assemblies. Front. Immunol. 8, 1407 (2017).

31. Yu, G. Y. et al. Cynomolgus macaque (Macaca fascicularis) immunoglobulin heavy chain locus description. Immunogenetics 68, 417-428 (2016) The authors of this paper used next-generation sequencing to examine the immunoglobulin loci of cynomolgus macaques and found similarities to the loci in humans; however, they found that the macaque immunoglobulin loci are more diverse than the human loci.

32. Sundling, $\mathrm{C}$. et al. Single-cell and deep sequencing of IgG-switched macaque B cells reveal a diverse Ig repertoire following immunization. J. Immunol. 192 3637-3644 (2014).

33. de Groot, N. G., Heijmans, C. M. C. \& Bontrop, R. E. AIDS in chimpanzees: the role of $\mathrm{MHC}$ genes. Immunogenetics 69, 499-509 (2017).

34. Letvin, N. L. et al. Acquired immunodeficiency syndrome in a colony of macaque monkeys. Proc. Natl Acad. Sci. USA 80, 2718-2722 (1983).

35. Daniel, M. D. et al. Isolation of T-cell tropic HTLV-III-like retrovirus from macaques. Science 228 1201-1204 (1985).

36. King, N. W., Hunt, R. D. \& Letvin, N. L. Histopathologic changes in macaques with an acquired immunodeficiency syndrome (AIDS). Am. J. Pathol. 113, 382-388 (1983).

37. Hirsch, V. M. et al. Molecular cloning of SIV from sooty mangabey monkeys. J. Med. Primatol. 18 279-285 (1989).

38. Hirsch, V. M., Olmsted, R. A., Murphey-Corb, M., Purcell, R. H. \& Johnson, P. R. An African primate lentivirus (SIVsm) closely related to HIV-2. Nature 339, 389-392 (1989)

39. Sharp, P. M., Robertson, D. L. \& Hahn, B. H. Crossspecies transmission and recombination of 'AIDS' viruses. Phil. Trans. R. Soc. Lond. B 349, 41-47 (1995).

40. Makori, N. et al. Functional and morphological development of lymphoid tissues and immune regulatory and effector function in rhesus monkeys cytokine-secreting cells, immunoglobulin-secreting cells, and CD5 $5^{+}$B-1 cells appear early in fetal development. Clin. Diagn. Lab. Immunol. 10 140-153 (2003).

41. Abel, K. The rhesus macaque pediatric SIV infection model - a valuable tool in understanding infant HIV-1 pathogenesis and for designing pediatric HIV-1 prevention strategies. Curr. HIV Res. 7, 2-11 (2009).

42. Miller, C. J. et al. Genital mucosal transmission of simian immunodeficiency virus: animal model for heterosexual transmission of human immunodeficiency virus. J. Virol. 63, 4277-4284 (1989).

43. Sodora, D. L., Gettie, A., Miller, C. J. \& Marx, P. A. Vaginal transmission of SIV: assessing infectivity and hormonal influences in macaques inoculated with cell-free and cell-associated viral stocks. AIDS Res. Hum. Retroviruses 14, (Suppl. 1). S119-S123 (1998).

44. Li, Q. et al. Glycerol monolaurate prevents mucosal SIV transmission. Nature 458, 1034-1038 (2009).

45. Keele, B. F. et al. Low-dose rectal inoculation of rhesus macaques by SIVsmE660 or SIVmac251 recapitulates human mucosal infection by HIV-1. J. Exp. Med. 206 1117-1134 (2009).

46. Ma, Z. M. et al. SIVmac251 is inefficiently transmitted to rhesus macaques by penile inoculation with a single SIVenv variant found in ramp-up phase plasma. AIDS Res. Hum. Retroviruses 27, 1259-1269 (2011). 
47. Barouch, D. H. et al. Rapid inflammasome activation following mucosal SIV infection of rhesus monkeys. Cell 165, 656-667 (2016).

48. Miller, C. J. Localization of Simian immunodeficiency virus-infected cells in the genital tract of male and female Rhesus macaques. J. Reprod. Immunol. $\mathbf{4 1}$ 331-339 (1998)

49. Zhang, Z. et al. Sexual transmission and propagation of SIV and HIV in resting and activated $\mathrm{CD}^{+} \mathrm{T}$ cells. Science 286, 1353-1357 (1999).

50. Miller, C. J. et al. Propagation and dissemination of infection after vaginal transmission of simian immunodeficiency virus. J. Virol. 79, 9217-9227 (2005).

This study demonstrates that, following vaginal challenge, an initial small founder viral population establishes infection within the mucosa prior to continuous seeding into draining lymphoid tissues and subsequently to systemic sites, which establishes a productive infection. This finding suggests that a small window of vulnerability exists to prevent or control infection.

51. Haase, A. T. Early events in sexual transmission of HIV and SIV and opportunities for interventions. Annu. Rev. Med. 62, 127-139 (2011)

52. Fennessey, C. M. \& Keele, B. F. Using nonhuman primates to model HIV transmission. Curr. Opin. HIV AIDS 8, 280-287 (2013).

53. Liu, J. et al. Low-dose mucosal simian immunodeficiency virus infection restricts early replication kinetics and transmitted virus variants in rhesus monkeys. J. Virol. 84, 10406-10412 (2010)

54. Lee, F. H. et al. Breakthrough virus neutralization resistance as a correlate of protection in a nonhuman primate heterologous simian immunodeficiency virus vaccine challenge study. J. Virol. 89, 12388-12400 (2015).

55. Gach, J. S. et al. Relationship between vaccine-induced antibody capture of infectious virus and infection outcomes following repeated low-dose rectal challenges with simian immunodeficiency virus SIVmac251. J. Virol. 90, 8487-8495 (2016)

56. Roederer, M. et al. Immunological and virological mechanisms of vaccine-mediated protection against SIV and HIV. Nature 505, 502-508 (2014).

57. Keele, B. F. et al. Adenovirus prime, Env protein boost vaccine protects against neutralization-resistant SIVsmE660 variants in rhesus monkeys. Nat. Commun. 8, 15740 (2017).

58. Evans, D. T. \& Silvestri, G. Nonhuman primate models in AIDS research. Curr. Opin. HIV AIDS 8, 255-261 (2013)

59. Rerks-Ngarm, S. et al. Vaccination with ALVAC and AIDSVAX to prevent HIV- 1 infection in Thailand. $N$. Engl. J. Med. 361, 2209-2220 (2009).

60. Haynes, B. F. et al. Immune-correlates analysis of an HIV-1 vaccine efficacy trial. N. Engl. J. Med. 366 1275-1286 (2012).

This study identifies that IgG antibodies targeting the $\mathrm{V} 1 / \mathrm{V} 2$ region of the HIV envelope protein may have contributed to protection against HIV-1 infection, whereas high levels of envelope-specific IgA antibodies in the plasma may have mitigated the effects of the protective IgG V1/V2 antibodies.

61. Pegu, P. et al. Antibodies with high avidity to the gp 120 envelope protein in protection from simian immunodeficiency virus SIV(mac251) acquisition in an immunization regimen that mimics the RV-144 Thai trial. J. Virol. 87, 1708-1719 (2013)

62. Putkonen, P. et al. Prevention of HIV-2 and SIVsm infection by passive immunization in cynomolgus monkeys. Nature 352, 436-438 (1991).

63. Van Rompay, K. K. et al. Passive immunization of newborn rhesus macaques prevents oral simian immunodeficiency virus infection. J. Infect. Dis. 177 1247-1259 (1998)

64. Shibata, R. et al. Neutralizing antibody directed against the HIV-1 envelope glycoprotein can completely block HIV-1/SIV chimeric virus infections of macaque monkeys. Nat. Med 5, 204-210 (1999).

65. Mascola, J. R. et al. Protection of macaques against vaginal transmission of a pathogenic HIV-1/SIV chimeric virus by passive infusion of neutralizing antibodies. Nat. Med 6, 207-210 (2000).

66. Hessell, A. J. et al. Fc receptor but not complement binding is important in antibody protection against HIV. Nature 449, 101-104 (2007)

67. Kelsoe, G. \& Haynes, B. F. Host controls of HIV broadly neutralizing antibody development. Immunol. Rev. 275, 79-88 (2017).
68. Shiver, J. W. et al. Replication-incompetent adenoviral vaccine vector elicits effective antiimmunodeficiency-virus immunity. Nature $\mathbf{4 1 5}$ 331-335 (2002)

69. McMichael, A. J. \& Koff, W. C. Vaccines that stimulate T cell immunity to HIV-1: the next step. Nat. Immunol. 15, 319-322 (2014).

70. Kumar, N., Chahroudi, A. \& Silvestri, G. Animal models to achieve an HIV cure. Curr. Opin. HIV AIDS 11, 432-441 (2016)

71. Brenchley, J. M. \& Douek, D. C. Microbial translocation across the Gl tract. Annu. Rev. Immunol. 30, 149-173 (2012)

72. Deeks, S. G. et al. Immune activation set point during early HIV infection predicts subsequent $\mathrm{CD}^{+} \mathrm{T}$-cell changes independent of viral load. Blood 104, 942-947 (2004).

73. Giorgi, J. V. et al. Shorter survival in advanced human immunodeficiency virus type 1 infection is more closely associated with $\mathrm{T}$ lymphocyte activation than with plasma virus burden or virus chemokine coreceptor usage. J. Infect. Dis. 179, 859-870 (1999).

74. Silvestri, G., Paiardini, M., Pandrea, I., Lederman M. M. \& Sodora, D. L. Understanding the benign nature of SIV infection in natural hosts. J. Clin. Invest. 117, 3148-3154 (2007)

75. Douek, D. C., Roederer, M. \& Koup, R. A. Emerging concepts in the immunopathogenesis of AIDS. Annu. Rev. Med. 60, 471-484 (2009).

76. Estes, J. D. Pathobiology of HIV/SIV-associated changes in secondary lymphoid tissues. Immunol. Rev. 254, 65-77 (2013).

77. Brenchley, J. M. et al. Microbial translocation is a cause of systemic immune activation in chronic HIV infection. Nat. Med. 12, 1365-1371 (2006).

78. Ciccone, E. J. et al. Cycling of gut mucosal CD4+ T cells decreases after prolonged anti-retroviral therapy and is associated with plasma LPS levels. Mucosal Immunol. 3, 172-181 (2010).

79. Jiang, W. et al. Plasma levels of bacterial DNA correlate with immune activation and the magnitude of immune restoration in persons with antiretroviralTreated HIV infection. J. Infect. Dis. 199, 1177-1185 (2009).

80. Estes, J. D. et al. Damaged intestinal epithelial integrity linked to microbial translocation in pathogenic simian immunodeficiency virus infections. PLoS Pathog. 6, e1001052 (2010).

81. Pandrea, I. V. et al. Acute loss of intestinal $\mathrm{CD}^{+}{ }^{+} \mathrm{T}$ cells is not predictive of simian immunodeficiency virus virulence. J. Immunol. 179, 3035-3046 (2007).

82. Hao, X. P. et al. Experimental colitis in SIV-uninfected rhesus macaques recapitulates important features of pathogenic SIV infection. Nat. Commun. 6, 8020 (2015).

This study reports the first NHP model of experimental colitis and provides definitive evidence that gastrointestinal tract damage, which leads to local and systemic microbial translocation, and the associated immune activation are important independent determinants of AIDS pathogenesis.

83. Chalifoux, L. V. et al. Lymphadenopathy in macaques experimentally infected with the simian immunodeficiency virus (SIV). Am. J. Pathol. 128, 104-110 (1987)

84. Schacker, T. W. et al Collagen deposition in HIV-1 infected lymphatic tissues and T cell homeostasis J. Clin. Invest. 110, 1133-1139 (2002)

85. Nilsson, J. et al. HIV-1-driven regulatory T-cell accumulation in lymphoid tissues is associated with disease progression in HIV/AIDS. Blood 108 3808-3817 (2006)

86. Andersson, J. et al. The prevalence of regulatory $T$ cells in lymphoid tissue is correlated with vira load in HIV-infected patients. J. Immunol. 174 3143-3147 (2005).

87. Lawitz, E. et al. Sofosbuvir and ledipasvir fixed-dose combination with and without ribavirin in treatment naive and previously treated patients with genotype 1 hepatitis $C$ virus infection (LONESTAR): an open-label, randomised, phase 2 trial. Lancet 383, 515-523 (2014).

88. Rockstroh, J. K. et al. Ledipasvir-sofosbuvir for 6 weeks to treat acute hepatitis $C$ virus genotype 1 or 4 infection in patients with HIV coinfection: an openlabel, single-arm trial. Lancet Gastroenterol. Hepatol 2, 347-353 (2017)

89. Bukh, J. A critical role for the chimpanzee model in the study of hepatitis C. Hepatology 39, 1469-1475 (2004).
90. Houghton, M. \& Abrignani, S. Prospects for a vaccine against the hepatitis C virus. Nature 436, 961-966 (2005).

91. Catanese, M. T. $\&$ Dorner, M. Advances in experimental systems to study hepatitis $C$ virus in vitro and in vivo. Virology 479-480, 221-233 (2015).

92. Lanford, R. E., Walker C. M. \& Lemon, S. M. The chimpanzee model of viral hepatitis: advances in understanding the immune response and treatment of viral hepatitis. ILAR J. 58, 172-189 (2017).

93. Farci, P. et al. The natural history of infection with hepatitis $\mathrm{C}$ virus $(\mathrm{HCV})$ in chimpanzees: comparison of serologic responses measured with first- and second-generation assays and relationship to $\mathrm{HCV}$ viremia. J. Infect. Dis. 165, 1006-1011 (1992). This is the first report of experimental transmission of an HCV strain from an infected individual with fulminant hepatic failure into a chimpanzee, which caused unusually severe acute hepatitis in the animal.

94. Farci, P. et al. Experimental transmission of hepatitis $\mathbf{C}$ virus-associated fulminant hepatitis to a chimpanzee. J. Infect. Dis. 179, 1007-1011 (1999).

95. Major, M. E. et al. Hepatitis C virus kinetics and host responses associated with disease and outcome of infection in chimpanzees. Hepatology 39 , 1709-1720 (2004)

96. Manickam, C. \& Reeves, R. K. Modeling HCV disease 'in animals: virology, immunology and pathogenesis of HCV and GBV-B infections. Front. Microbiol 5, 690 (2014).

97. Smith, D. B. et al. Proposed reference sequences for hepatitis E virus subtypes. J. Gen. Virol. 97, 537-542 (2016).

98. Bradley, D. W. et al. Enterically transmitted non-A, non$B$ hepatitis: serial passage of disease in cynomolgus macaques and tamarins and recovery of diseaseassociated 27- to 34-nm viruslike particles. Proc. Natl Acad. Sci. USA 84, 6277-6281 (1987).

This study reports the successful generation of an NHP model of HEV infection by performing serial passages of enterically derived human non-A, non-B hepatitis viral particles into cynomolgus macaques.

99. Kane, M. A. et al. Epidemic non-A, non-B hepatitis in Nepal. Recovery of a possible etiologic agent and transmission studies in marmosets. JAMA 252 3140-3145 (1984).

100. Arankalle, V. A., Chobe, L. P. \& Chadha, M. S. Type-IV Indian swine HEV infects rhesus monkeys. J. Viral Hepat. 13, 742-745 (2006).

101. van Cuyck-Gandre, H. et al. Experimental African HEV infection in cynomolgus macaques (Macaca fascicularis). J. Med. Virol. 55, 197-202 (1998).

102. McCaustland, K. A. et al. Hepatitis E virus infection in chimpanzees: a retrospective analysis. Arch. Virol. 145, 1909-1918 (2000).

103. Tsarev, S. A. et al. Variation in course of hepatitis $E$ in experimentally infected cynomolgus monkeys. J. Infect. Dis. 167, 1302-1306 (1993).

104. Tsarev, S. A. et al. Experimental hepatitis E in pregnant rhesus monkeys: failure to transmit hepatitis $\mathrm{E}$ virus (HEV) to offspring and evidence of naturally acquired antibodies to HEV. J. Infect. Dis. 172, 31-37 (1995).

105. Zhang, J. et al. Evaluation of antibody-based and nucleic acid-based assays for diagnosis of hepatitis $E$ virus infection in a rhesus monkey model. J. Med. Virol. 71, 518-526 (2003)

106. Aggarwal, R., Kamili, S., Spelbring, J. \& Krawczynski, K. Experimental studies on subclinical hepatitis E virus infection in cynomolgus macaques. J. Infect. Dis. 184 1380-1385 (2001)

107. Purcell, R. H. et al. Pathobiology of hepatitis E: lessons learned from primate models. Emerg. Microbes Infect. 2, e9 (2013).

108. Purcell, R. H. \& Emerson, S. U. Animal models of hepatitis A and E. ILAR J. 42, 161-177 (2001)

109. Li, S. W. et al. A bacterially expressed particulate hepatitis E vaccine: antigenicity, immunogenicity and protectivity on primates. Vaccine 23, 2893-2901 (2005).

110. Purcell, R. H. et al. Pre-clinical immunogenicity and efficacy trial of a recombinant hepatitis $E$ vaccine. Vaccine 21, 2607-2615 (2003)

111. Tsarev, S. A. et al. Successful passive and active immunization of cynomolgus monkeys against hepatitis E. Proc. Natl Acad. Sci. USA 91 10198-10202 (1994).

112. Tsarev, S. A. et al. Recombinant vaccine against hepatitis $\mathrm{E}$ : dose response and protection agains 
heterologous challenge. Vaccine 15, 1834-1838 (1997).

113. Zhang, M. et al. Immunogenicity and protective efficacy of a vaccine prepared from $53 \mathrm{kDa}$ truncated hepatitis $\mathrm{E}$ virus capsid protein expressed in insect cells. Vaccine 20, 853-857 (2001).

114. Shrestha, M. P. et al. Safety and efficacy of a recombinant hepatitis E vaccine. N. Engl. J. Med. 356 895-903 (2007)

115. Pierson, T. C. \& Kielian, M. Flaviviruses: braking the entering. Curr. Opin. Virol. 3, 3-12 (2013).

116. Samuel, M. A. \& Diamond, M. S. Alpha/beta interferon protects against lethal West Nile virus infection by restricting cellular tropism and enhancing neuronal survival. J. Virol. 79, 13350-13361 (2005).

117. Meier, K. C., Gardner, C. L., Khoretonenko, M. V., Klimstra, W. B. \& Ryman, K. D. A mouse model for studying viscerotropic disease caused by yellow fever virus infection. PLoS Pathog. 5, e1000614 (2009).

118. Morrison, T. E. \& Diamond, M. S. Animal Models of Zika virus infection, pathogenesis, and immunity. J. Virol. 91, e00009-17 (2017).

119. Clark, K. B., Onlamoon, N., Hsiao, H. M., Perng G. C. \& Villinger, F. Can non-human primates serve as models for investigating dengue disease pathogenesis? Front. Microbiol 4, 305 (2013)

120. Fox, A. et al. Immunological and viral determinants of dengue severity in hospitalized adults in $\mathrm{Ha} \mathrm{Noi}$, Viet Nam. PLoS Negl. Trop. Dis. 5, e967 (2011).

121. Sariol, C. A. \& White, L. J. Utility, limitations, and future of non-human primates for dengue research and vaccine development. Front. Immunol. 5, 452 (2014).

122. Durbin, A. P., Kirkpatrick, B. D., Pierce, K. K., Schmidt, A. C. \& Whitehead, S. S. Development and clinical evaluation of multiple investigational monovalent DENV vaccines to identify components for inclusion in a live attenuated tetravalent DENV vaccine. Vaccine 29, 7242-7250 (2011)

123. Whitehead, S. S. et al. In a randomized trial, the live attenuated tetravalent dengue vaccine TV003 is welltolerated and highly immunogenic in subjects with flavivirus exposure prior to vaccination. PLoS Negl. Trop. Dis. 11, e0005584 (2017).

124. Kirkpatrick, B. D. et al. The live attenuated dengue vaccine TV003 elicits complete protection against dengue in a human challenge model. Sci. Transl Med. 8, 330 ra336 (2016).

125. Baud, D., Gubler, D. J., Schaub, B., Lanteri, M. C. \& Musso, D. An update on Zika virus infection. Lancet 390, 2099-2109 (2017).

126. Li, X. F. et al. Characterization of a 2016 clinical isolate of Zika virus in non-human primates. EBioMedicine 12, 170-177 (2016)

127. Dudley, D. M. et al. A rhesus macaque model of Asianlineage Zika virus infection. Nat. Commun. 7, 12204 (2016).

128. Osuna, C. E. et al. Zika viral dynamics and shedding in rhesus and cynomolgus macaques. Nat. Med. 22 1448-1455 (2016)

129. Nguyen, S. M. et al. Highly efficient maternal-fetal Zika virus transmission in pregnant rhesus macaques. PLoS Pathog. 13, e1006378 (2017).

130. Abbink, P. et al. Protective efficacy of multiple vaccine platforms against Zika virus challenge in rhesus monkeys. Science 353, 1129-1132 (2016).

131. Dowd, K. A. et al. Rapid development of a DNA vaccine for Zika virus. Science 354, 237-240 (2016). Shortly after the ZIKV epidemic began, this study developed several vaccine modalities and showed that DNA vaccination could be a successful approach to protect against ZIKV infection in NHPs.

132. Larocca, R. A. et al. Vaccine protection against Zika virus from Brazil. Nature 536, 474-478 (2016).

133. Driggers, R. W. et al. Zika virus infection with prolonged maternal viremia and fetal brain abnormalities. N. Engl. J. Med. 374, 2142-2151 (2016).

134. Koide, F. et al. Development of a Zika virus infection model in cynomolgus macaques. Front. Microbiol. 7 2028 (2016)

135. Hirsch, A. J. et al. Zika Virus infection of rhesus macaques leads to viral persistence in multiple tissues. PLoS Pathog. 13, e1006219 (2017).

136. Yeung, M. W., Shing, E., Nelder, M. \& Sander, B. Epidemiologic and clinical parameters of West Nile virus infections in humans: a scoping review. $B M C$ Infect. Dis. 17, 609 (2017).

137. Verstrepen, B. E. et al. Experimental infection of rhesus macaques and common marmosets with a
European strain of West Nile virus. PLoS Negl. Trop. Dis. 8, e2797 (2014)

138. Maximova, O. A., Bernbaum, J. G. \& Pletnev, A. G. West Nile virus spreads transsynaptically within the pathways of motor control: anatomical and ultrastructural mapping of neuronal virus infection in the primate central nervous system. PLoS Negl. Trop. Dis. 10, e0004980 (2016).

139. Lieberman, M. M. et al. Immunogenicity and protective efficacy of a recombinant subunit West Nile virus vaccine in rhesus monkeys. Clin. Vaccine Immunol. 16, 1332-1337 (2009).

140. Verstrepen, B. E. et al. Vaccine-induced protection of rhesus macaques against plasma viremia after intradermal infection with a European lineage 1 strain of West Nile virus. PLOS ONE 9, e 112568 (2014).

141. Arroyo, J. et al. ChimeriVax-West Nile virus liveattenuated vaccine: preclinical evaluation of safety, immunogenicity, and efficacy. J. Virol. 78, 12497-12507 (2004).

142. Widman, D. G. et al. Evaluation of RepliVAX WN, a single-cycle flavivirus vaccine, in a non-human primate model of West Nile virus infection. Am. J. Trop. Med. Hyg. 82, 1160-1167 (2010)

143. Ouwendijk, W. J. \& Verjans, G. M. Pathogenesis of varicelloviruses in primates. J. Pathol. 235 298-311 (2015).

144. Haberthur, K. et al. CD4 T cell immunity is critical for the control of simian varicella virus infection in a nonhuman primate model of VZV infection. PLoS Pathog. 7, e1002367 (2011).

This study is the first to demonstrate that CD4 T cells are important for controlling VZV-like virus infection and spread.

145. Traina-Dorge, V. et al. Simian varicella virus is present in macrophages, dendritic cells, and T cells in lymph nodes of rhesus macaques after experimental reactivation. J. Virol. 89, 9817-9824 (2015).

146. Itell, H. L., Kaur, A., Deere, J. D., Barry, P. A. \& Permar, S. R. Rhesus monkeys for a nonhuman primate model of cytomegalovirus infections. Curr. Opin. Virol. 25, 126-133 (2017).

147. Pitcher, C. J. et al. Development and homeostasis of $\mathrm{T}$ cell memory in rhesus macaque. J. Immunol. 168 29-43 (2002).

This study is the first to define rhesus macaque T cell subsets and characterize their functional phenotypes.

148. Hansen, S. G. et al. Effector memory T cell responses are associated with protection of rhesus monkeys from mucosal simian immunodeficiency virus challenge. Nat. Med. 15, 293-299 (2009)

149. Fruh, K. \& Picker, L. CD8 ${ }^{+}$T cell programming by cytomegalovirus vectors: applications in prophylactic and therapeutic vaccination. Curr. Opin. Immunol. 47 52-56 (2017).

150. Hansen, S. G. et al. Profound early control of highly pathogenic SIV by an effector memory T-cell vaccine. Nature 473, 523-527 (2011).

This study is the first to demonstrate that a $\mathrm{T}$ cell vaccine can protect rhesus macaques from a pathogenic SIV infection and informs how a human vaccine may be designed to prevent HIV infection.

151. Muhe, J. \& Wang, F. Non-human primate lymphocryptoviruses: past, present, and future. Curr. Top. Microbiol. Immunol. 391, 385-405 (2015).

152. Ohashi, M., Orlova, N., Quink, C. \& Wang, F. Cloning of the Epstein-Barr virus-related rhesus lymphocryptovirus as a bacterial artificial chromosome: a loss-of-function mutation of the rhBARF1 immune evasion gene. J. Virol. 85, 13301339 (2011)

153. Orlova, N., Fogg, M. H., Carville, A. \& Wang, F. Antibodies to lytic infection proteins in lymphocryptovirus-infected rhesus macaques: a mode for humoral immune responses to epstein-barr virus infection. Clin. Vaccine Immunol. 18, 1427-1434 (2011).

154. Moutschen, M. et al. Phase I/II studies to evaluate safety and immunogenicity of a recombinant gp350 Epstein-Barr virus vaccine in healthy adults. Vaccine 25, 4697-4705 (2007)

155. Sokal, E. M. et al. Recombinant gp350 vaccine for infectious mononucleosis: a phase 2 , randomized double-blind, placebo-controlled trial to evaluate the safety, immunogenicity, and efficacy of an Epstein-Bar virus vaccine in healthy young adults. J. Infect. Dis. 196, 1749-1753 (2007)

156. Herrman, M., Muhe, J., Quink, C. \& Wang, F. EpsteinBarr virus gp350 can functionally replace the rhesus lymphocryptovirus major membrane glycoprotein and does not restrict infection of rhesus macaques. J. Virol. 90, 1222-1230 (2015)

This study demonstrates that EBV gp350 is capable of functionally substituting for rhesus LCV gp350 and that infection of rhesus macaques with recombinant rhLCV could prove valuable in the evaluation of novel vaccine approaches to prevent EBV infection.

157. Leskowitz, R. et al. Adenovirus-based vaccines against rhesus lymphocryptovirus EBNA-1 induce expansion of specific $\mathrm{CD} 8^{+}$and $\mathrm{CD} 4^{+} \mathrm{T}$ cells in persistently infected rhesus macaques. J. Virol. 88, 4721-4735 (2014).

158. Leskowitz, R. M. et al. CD4+ and CD8+ T-cell responses to latent antigen EBNA-1 and lytic antigen BZLF-1 during persistent lymphocryptovirus infection of rhesus macaques. J. Virol. 87, 8351-8362 (2013).

159. Russo, J. J. et al. Nucleotide sequence of the Kaposi sarcoma-associated herpesvirus (HHV8). Proc. Natl Acad. Sci. USA 93, 14862-14867 (1996).

160. Cesarman, E., Chang, Y., Moore, P. S., Said, J. W. \& Knowles, D. M. Kaposi's sarcoma-associated herpesvirus-like DNA sequences in AIDS-related body-cavity-based lymphomas. N. Engl. J. Med. 332, 1186-1191 (1995).

161. Soulier, J. et al. Kaposi's sarcoma-associated herpesvirus-like DNA sequences in multicentric Castleman's disease. Blood 86, 1276-1280 (1995).

162. Chang, H. et al. Non-human primate model of Kaposi's sarcoma-associated herpesvirus infection. PLoS Pathog. 5, e1000606 (2009).

163. Desrosiers, R. C. et al. A herpesvirus of rhesus monkeys related to the human Kaposi's sarcomaassociated herpesvirus. J. Virol. 71, 9764-9769 (1997).

164. Wong, S. W. et al. Induction of B cell hyperplasia in simian immunodeficiency virus-infected rhesus macaques with the simian homologue of Kaposi's sarcoma-associated herpesvirus. J. Exp. Med. 190 827-840 (1999).

165. Mansfield, K. G. et al. Experimental infection of rhesus and pig-tailed macaques with macaque rhadinoviruses. J. Virol. 73, 10320-10328 (1999).

166. Orzechowska, B. U. et al. Rhesus macaque rhadinovirus-associated non-Hodgkin lymphoma: animal model for KSHV-associated malignancies. Blood 112, 4227-4234 (2008).

167. Estep, R. D. \& Wong, S. W. Rhesus macaque rhadinovirus-associated disease. Curr. Opin. Virol. 3, 245-250 (2013)

168. Robinson, B. A. et al. Viral interferon regulatory factors are critical for delay of the host immune response against rhesus macaque rhadinovirus infection. J. Virol. 86, 2769-2779 (2012). This study demonstrates how viral-encoded proteins that are postulated to inhibit the interferon response can impact the host response to virus infection in vivo.

169. Nakayama, E. \& Saijo, M. Animal models for Ebola and Marburg virus infections. Front. Microbiol. 4, 267 (2013).

170. Warren, T. K. et al. Therapeutic efficacy of the small molecule GS-5734 against Ebola virus in rhesus monkeys. Nature 531, 381-385 (2016). The authors of this paper found a small-molecularmass compound that could reduce replication of Ebola virus in NHPs. These data could lead to nove therapeutic interventions to reduce filovirus epidemics.

171. Misasi, J. et al. Structural and molecular basis for Ebola virus neutralization by protective human antibodies. Science 351, 1343-1346 (2016).

172. Corti, D. et al. Protective monotherapy against lethal Ebola virus infection by a potently neutralizing antibody. Science 351, 1339-1342 (2016).

173. Mire, C. E. et al. Therapeutic treatment of Marburg and Ravn virus infection in nonhuman primates with a human monoclonal antibody. Sci. Transl Med. 9, eaai8711 (2017)

174. Earl, P. L. et al. Immunogenicity of a highly attenuated MVA smallpox vaccine and protection against monkeypox. Nature 428, 182-185 (2004).

175. Edghill-Smith, Y. et al. Smallpox vaccine-induced antibodies are necessary and sufficient for protection against monkeypox virus. Nat. Med. 11, 740-747 (2005).

176. Stittelaar, K. J. et al. Antiviral treatment is more effective than smallpox vaccination upon lethal monkeypox virus infection. Nature 439, 745-748 (2006). 
177. Labadie, K. et al. Chikungunya disease in nonhuman primates involves long-term viral persistence in macrophages. J. Clin. Invest. 120, 894-906 (2010).

178. Messaoudi, I. et al. Chikungunya virus infection results in higher and persistent viral replication in aged rhesus macaques due to defects in anti-viral immunity. PLoS Negl. Trop. Dis. 7, e2343 (2013).

179. Roy, C. J. et al. Chikungunya vaccine candidate is highly attenuated and protects nonhuman primates against telemetrically monitored disease following a single dose. J. Infect. Dis. 209, 1891-1899 (2014).

180. Mallilankaraman, K. et al. A DNA vaccine against chikungunya virus is protective in mice and induces neutralizing antibodies in mice and nonhuman primates. PLoS Negl. Trop. Dis. 5, e928 (2011)

181. Margulies, E. H. et al. Analyses of deep mammalian sequence alignments and constraint predictions for $1 \%$ of the human genome. Genome Res. 17, 760-774 (2007)

\section{Acknowledgements}

Funding for this work was provided in part with federal funds from the US Division of Intramural Research, National
Institute of Allergy and Infectious Diseases, National Institutes of Health $(\mathrm{NIH})$ and the National Cancer Institute, NIH, under contract number HHSN261200800001E and NIH grants P51-OD011092 (S.W.W. and J.D.E.), R01-CA75922 (S.W.W. and R01-CA206404 (S.W.W.). The content of this publication does not necessarily reflect the views or policies of the US Department of Health and Human Services, nor does the mention of trade names, commercial products or organizations imply endorsement by the US government.

\section{Author contributions}

The authors wrote the article together and all authors contributed to research, discussions, review and editing.

\section{Competing interests}

The authors declare no competing interests

Publisher's note

Springer Nature remains neutral with regard to jurisdictiona claims in published maps and institutional affiliations.

\section{Reviewer information}

Nature Reviews Immunology thanks Peter A. Barry, Tomás Hanke and the other anonymous reviewer(s) for their contribution to the peer review of this work.

\section{RELATED LINKS}

NIH Office of Research Infrastructure Programs -

Comparative Medicine - Vertebrate Models:

https://orip.nih.gov/comparative-medicine/programs/ vertebrate-models

National Primate Research Centers:

https://nprcresearch.org/primate/

Biomedical Primate Research Centre:

http://www.bprc.nl/en/home/

NIH NHP Reagent Resource:

http://www.nhpreagents.org/NHP/default.aspx

NHP MHC web portal:

https://dholk.primate.wisc.edu/_webdav/dho/mhc_contract/

web_portal/\%40files/prototype/index.html

Immuno Polymorphism Database (IPD) - MHC:

https://www.ebi.ac.uk/ipd/mhc/group/NHP

The Macaque Genotype and Phenotype Database:

https://mgap.ohsu.edu/

NHP Genome Sequences:

https://www.hysc.bcm.edu/non-human-primates

NHP Reference Transcriptome Resource:

http://nhprtr.org/

Virus Pathogen Database and Analysis Resource:

https://www.viprbrc.org 